\title{
Trimethylsilyl chloride promoted synthesis of a-branched amines by nucleophilic addition of organozinc halides to nitrones
}

Fu, Ying; Liu, Yanhua; Chen, Yaojuan; Hugel, Helmut; Wang, Minzhu; Huang, Danfeng; Hu, Yulai https://researchrepository.rmit.edu.au/esploro/outputs/9921858632001341/filesAndLinks?institution=61RMIT_INST\&index=null

Fu, Y., Liu, Y., Chen, Y., Hugel, H., Wang, M., Huang, D., \& Hu, Y. (2012). Trimethylsilyl chloride promoted synthesis of a-branched amines by nucleophilic addition of organozinc halides to nitrones. Organic and Biomolecular Chemistry, 10(38), 7669-7672. https://doi.org/10.1039/c2ob26202a

Document Version: Accepted Manuscript

Published Version: https://doi.org/10.1039/c2ob26202a

Repository homepage: https://researchrepository.rmit.edu.au (c) 2012 R S C Publications

Downloaded On 2023/04/27 00:49:50 +1000 
Thank you for downloading this document from the RMIT Research Repository.

The RMIT Research Repository is an open access database showcasing the research outputs of RMIT University researchers.

RMIT Research Repository: http://researchbank.rmit.edu.au/

\section{Citation:}

Fu, Y, Liu, Y, Chen, Y, Hugel, H, Wang, M, Huang, D and Hu, Y 2012, 'Trimethylsilyl chloride promoted synthesis of a-branched amines by nucleophilic addition of organozinc halides to nitrones', Organic and Biomolecular Chemistry, vol. 10, no. 38, pp. 7669-7672.

See this record in the RMIT Research Repository at:

http://researchbank.rmit.edu.au/view/rmit:18132

Version: Accepted Manuscript

Copyright Statement: (c) 2012 R S C Publications

Link to Published Version:

http://dx.doi.org/10.1039/c2ob26202a 


\title{
Trimethylsilyl Chloride Promoted Synthesis of $\alpha$-branched amines by Nucleophilic Addition of Organozinc Halides to Nitrones
}

\author{
Ying Fu, ${ }^{* a}$ Yanhua Liu, ${ }^{a}$ Yaojuan Chen, ${ }^{a}$ Helmut M. Hügel, ${ }^{b}$ Danfeng Huang ${ }^{a}$ and Yulai Hu ${ }^{a}$ \\ Received (in $X X X, X X X)$ Xth $X X X X X X X X X 20 X X$, Accepted Xth $X X X X X X X X X 20 X X$ \\ ${ }_{5}$ DOI: 10.1039/b000000x
}

A general procedure for the nucleophilic addition of organiczinc halides with nitrones in the presence of trimethylsilyl chloride has been developed. Trimethylsilyl chloride was found to be both an indispensable reaction ${ }_{10}$ promoter and a ready hydroxylamine protection agent in these reactions. The produced 0 (trimethylsilyl)hydroxylamines can be easily reduced into corresponding amines just by a zinc-copper couple in saturated aqueous $\mathrm{NH}_{4} \mathrm{Cl}$ solution.

$15 \alpha$-Branched amines represent an important class of nitrogen containg compounds regarding their attractive biological and pharmacological activities. As for example, Remacemide is used for the treatment of acute ischemic stroke, epilepsy, Huntington's disease and Parkinson's disease. ${ }^{1}$ Rivastigmine is 20 a reversible cholinesterase inhibitoror and is used to treat Alzheimer's disease. ${ }^{2}$ In addition, $\alpha$-Branched amines are highly valuable molecules in bioorganic chemistry as they are widely found in natral such as 2,5-dideoxy-2,5-imino- $D$ mannitol DMDP and 2,5-dideoxy-2,5-imino-DL-glycero-D-

25 manno-heptitol (homo-DMDP) which are found to be efficient glycosidas inhibitors. ${ }^{3}$ Thus, efficient syntheses of a library of $\alpha$-branched amines are of interest in both organic and medicinal chemistry. ${ }^{4}$

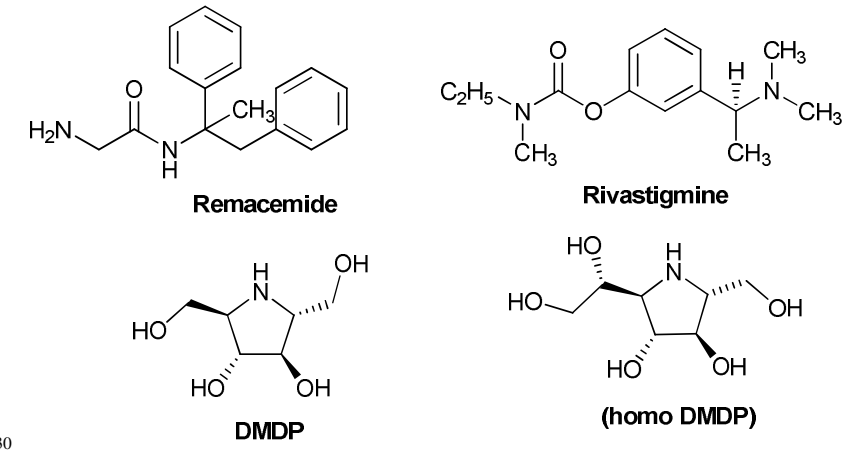

Fig 1. drugs and natural products of $\alpha$-branched amine

The nucleophilic addition reaction of organometallic reagents to nitrones is a convenient and reliable procedure, and hence, 35 is widely used in the synthesis of structurally variable $\alpha$ branched amines. ${ }^{5}$ Grignard reagents and organolithium reagents are frequently deployed in these conversions due to their ready availability and high reactivity, especially when chiral nitrones ${ }^{6}$ or chiral catalysts ${ }^{7}$ were introduced, high 40 yields as well as high stereoselectivities are always obtained. A large variety of natural and semi-natural products such as pyrrolizidines and indolizidines were successfully prepared via this protocol. ${ }^{8}$ However, Organozinc reagents, as a kind of less reactive yet more selective organometallic reagents. ${ }^{9}$ 45 although extensively used in the reactions with carbonyl compounds, are less explored in reactions with imine derivatives, such as nitrones. A few reports have appeared in which some reactive organozinc species, such as diorganozinc $^{10}$ or diorganozinc mediated alkynylation, ${ }^{11}$ 50 allylation, ${ }^{12}$ vinylation ${ }^{13}$ and the Reformasky reaction ${ }^{14}$ are effective. However, to the best of our knowledge, a practical synthesis of $\alpha$-branched amine derivatives by nucleophilic addition of nitrones with organozinc halides has not been reported to date.

55 Actually, organozinc halides are one of the most useful organometallic reagents that can be easily prepared by direct insertion of zinc metal into corresponding functionalized organic halides and are therefore considered as a "real kind of" functionalized organometallics. ${ }^{15}$ We were attracted by their wide 60 application in synthetic chemistry, and have reported that reaction of benzylic zinc halides with aryl aldehydes under transitionmetal-complex catalysis in the presence of trimethylsilyl chloride (TMSCl) leads to trans-stilbenes in high yield, ${ }^{16}$ whereas reaction of primary alkyl zinc halides with aromatic aldehydes under the ${ }_{65}$ same conditions gave $\alpha, \alpha$-dialkyltoluenes. ${ }^{17}$ To broaden the utility of organozinc reagents in organic synthesis, especially with the aim of exploring the utilization of organozinc halides as a mild and selectively functionalized nucleophile for the synthesis of $\alpha$ branched amines, we report here an interesting TMSCl promoted 70 nucleophilic addition reaction of organozinc halides with nitrones in which TMSCl was found to be both an indispensable reaction promoter and a ready hydroxylamine protection reagent.

Initially, the reaction of benzylzinc chloride 1a and $\mathrm{N}$-(4methoxybenzylidene)aniline oxide $\mathbf{2 a}$ was examined without any 75 catalyst. Unfortunately, no reaction occurred at room temperature. When the reaction temperature was raised to $60{ }^{\circ} \mathrm{C}$, the reaction proceeded sluggishly and after 6 hours, the nitrone $\mathbf{2 a}$ had completely reacted and the corresponding hydroxylamine 3a was obtained in $65 \%$ yield (Table 1, entries 1 and 2). 
Lewis acids can greatly enhance the reactivity of nitrones in various kinds of reactions such as 1,3-dipolar cycloaddition ${ }^{18}$ and nucleophilic addition reactions. ${ }^{8 \mathrm{~b}}$ When one equiv of TMSCl was added, the reaction proceeded slowly at room temperature and 5 nitrone 2a disappeared in six hours. However, hydroxylamine 3a and the O-TMS hydroxylamine ether $\mathbf{4 a}$ were formed in $78 \%$ overall yield (3a/4a 1:3). When 2 equivalents of TMSCl were added, the reaction proceeded quite quickly and cleanly. Nitrone 2a reacted in only 30 minutes and the O-TMS hydroxylamine 10 ether 4 a was obtained as the only product in $92 \%$ isolated yield (Table 1, entries 3 and 4).

With the preliminary success of the effect of trimethylsilyl chloride, several other organosilyl reagents were then screened. TMSBr, TMSI and TMSOTf worked in the same way in this 15 reaction system. They all gave the desired silylated hydroxylamine derivatives $\mathbf{4 a}$ cleanly with similar yield compared to TMSCl (entries 5-7). However, the widely used hydroxyl group protection reagents, tert-butyldimethylsilyl chloride (TBDMSCl) and tert-butyldiphenylsilyl chloride 20 (TBDPSCl) were ineffective (entries 8,9). When considering the price and availability of these trimethylsilyl compounds, TMSCl is the best choice and was selected in our following studies.

Table 1. Optimization of the reaction conditions ${ }^{a}$

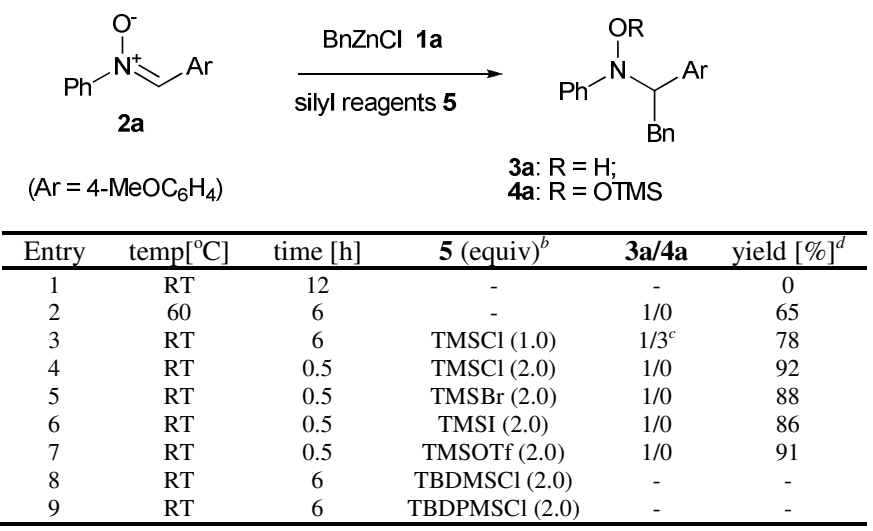

${ }_{25}{ }^{a}$ Reaction conditions: nitrone $\mathbf{2 a}(3 \mathrm{mmol})$ was treated with benzylzinc chloride 1a $(4.5 \mathrm{mmol})$ and organosilyl halides in THF $(15 \mathrm{~mL})$ at room temperature under argon. ${ }^{b}$ Amount of organosilyl halides was based on nitrone 2a. ${ }^{c}$ The ratio was determined by ${ }^{1} \mathrm{H}$ NMR spectra. ${ }^{d}$ Isolated yields.

$30 \quad$ Attracted by this unexpected reactivity, we then investigated the efficacy of TMSCl on reactions of various benzylic zinc halides and $\alpha, N$-diaryl nitrones. Different kinds of $\alpha, N$-diaryl nitrones bearing both electron donating and electron withdrawing groups were subjected to the optimized reaction conditions (Table 352 ). The results showed that the reaction has broad applicability. $\mathrm{N}$-phenyl nitrones bearing both electron-donating and electronwithdrawing groups on the C-phenyl rings reacted with benzylic zinc halide to give the corresponding $\mathrm{O}$-silylated hydroxylamines in high to very high yields (Table 2, entries 1-11). Aromatic 40 heterocyclic aldehydes derivated nitrones such as 3-thienyl and 2furanyl substituted nitrones all reacted smoothly with benzylic zinc halides with the corresponding silylated hydroxylamine derivatives were obtained in high (Table 2, entries 9, 10, 12 and 18). Interestingly, hydroxyl substituents on the benzylidine ring 45 of the nitrones do not require further protection as the desired $\mathrm{O}$ - silylated hydroxylamine ethers were obtained in good yield when a 2.5 molar ratio of benzylic zinc chloride was added (Table 2, entries 7 and 11).

Encouraged by our success with benzylic zinc chlorides, we 50 then investigated other organozinc reagents (Table 3). To our delight, other organozinc reagents $\left(\mathrm{R}^{1} \mathrm{ZnBr}\right)$ selected, such as $\mathrm{R}^{1}=$ aryl (entries 1-5, 15), hetero aromatics $\mathrm{R}^{1}=3$-thienyl (entries 6-8) and alkyl zincs (entries 9-14) all gave the desired products in high yields (entries 1-15). Alkyl zinc halide species of both primary 55 alkyl zinc halide (entries 9-12) and secondary alkyl zinc halide (entries 13,14) can also be applied here. Halogens such as chloro, fluoro are tolerated in both organozinc halides and nitrones. However, nitrones bearing hydroxyl groups when subjected to organozinc reagents, were unreactive (entries 16 and 17). This is 60 probably due to the strong electron donating effects of the phenolate, of the hydroxylated nitrone ${ }^{19}$ significantly reducing the electrophilicity of these nitrones so that only the more reactive organozinc species such as benzylic zinc halide can be used successfully.

${ }_{65}$ Table 2. Reaction of benzylic zinc chlorides with $\alpha, \mathrm{N}$-diaryl nitrones ${ }^{a}$

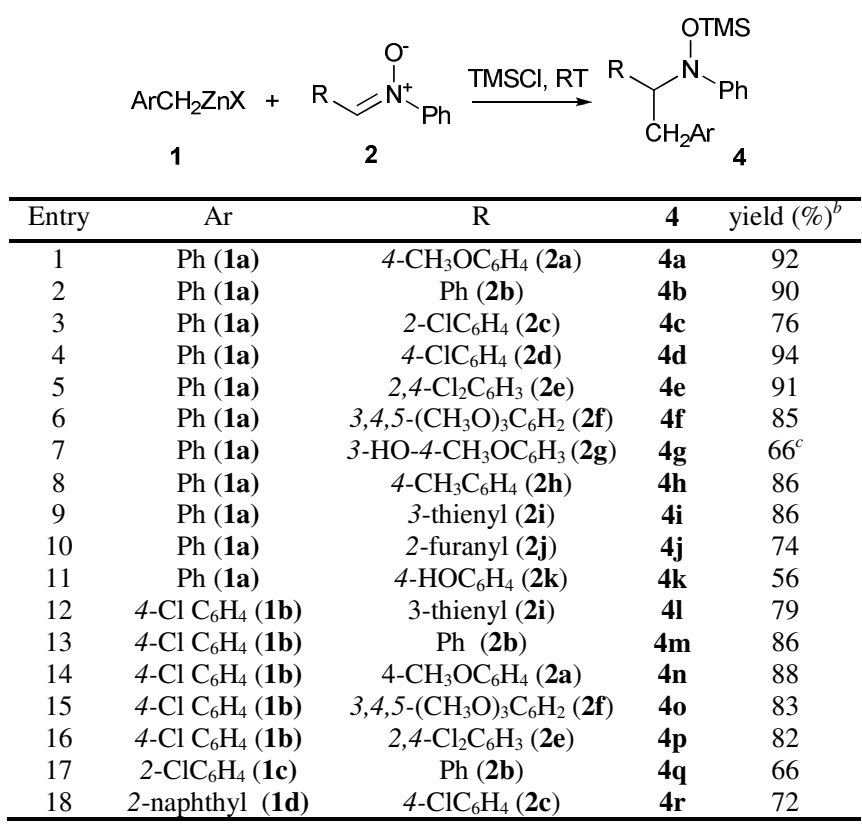

${ }^{a}$ Unless otherwise noted, the reaction was performed by employing benzylic zinc chloride $(4.5 \mathrm{mmol}), \mathrm{N}, \alpha$-diphenyl nitrone $(3 \mathrm{mmol})$ in THF (15 mL). ${ }^{b}$ Isolated yield. ${ }^{c}$ Benzylic zinc halide ( 2.5 equiv).

70 Although Grignard reagents can react readily with nitrones and give hydroxylamines in high yield, ${ }^{20}$ our $\mathrm{TMSCl}$ promoted organozinc reagent method has some significant advantages. The organozinc reagent can be highly functionalized whereas functionalized Grignard reagents are not readily utilized. The 75 most practical advantage of our protocol is that the reaction of Grignard reagents with nitrones leads only to hydroxylamines and since the hydroxylamine derivatives, especially $\mathrm{N}$-substituted aromatic hydroxylamines are very air sensitive, they decompose readily ${ }^{[20]}$ and usually cannot be isolated in a pure form by 80 quickly polymerizing after column chromatography. Thus, the synthesis, purification and characterization of hydroxylamines proved challenging. Even when these compounds were kept 
under nitrogen in a deep freezer for several weeks, they were found to have decomposed as indicated by ${ }^{1} \mathrm{H}$ NMR analysis. However, in our protocol, the $O$-TMS hydroxylamine ethers are stable to air and can be kept in a refrigerator for months without 5 any decomposition. We consider our protocol whereby $\mathrm{TMSCl}$ serves as a reaction promoter and a ready $N$-hydroxylamine protecting reagent is a significant improvement on the reaction of anyother previously reported Grignard and diorganozinc methods.

Table 3. Reaction of various organozinc halides with nitrones ${ }^{a}$

\begin{tabular}{|c|c|c|c|c|c|}
\hline $\begin{array}{r}\mathrm{R}_{1} \mathrm{ZnX} \\
7\end{array}$ & + & $={ }^{\stackrel{\mathrm{O}^{-}}{\mathrm{N}^{+}}}$ & $\frac{\text { TMSC }}{\text { RT }}$ & $\prod_{\mathrm{R}_{1}}^{\mathrm{R}_{2}} \overbrace{\mathbf{6 a} \sim \mathrm{n}}^{\mathrm{O}}$ & \\
\hline Entry & $\overline{6}$ & $\mathrm{R}^{1}$ & $\mathrm{R}^{2}$ & $\overline{\mathrm{R}^{3}}$ & $\operatorname{yield}(\%)^{b}$ \\
\hline 1 & $6 \mathbf{a}$ & $\mathrm{Ph}$ & $\mathrm{Ph}$ & $4-\mathrm{FC}_{6} \mathrm{H}_{4}$ & 84 \\
\hline 2 & $\mathbf{6 b}$ & $\mathrm{Ph}$ & $\mathrm{Ph}$ & $2-\mathrm{ClC}_{6} \mathrm{H}_{4}$ & 71 \\
\hline 3 & 6c & $\mathrm{Ph}$ & $\mathrm{Ph}$ & $3,4,5-\left(\mathrm{CH}_{3} \mathrm{O}\right)_{3} \mathrm{C}_{6} \mathrm{H}_{2}$ & 91 \\
\hline 4 & 6d & $\mathrm{Ph}$ & $\mathrm{Bn}$ & $\mathrm{Ph}$ & 88 \\
\hline 5 & $6 \mathbf{b}$ & $2-\mathrm{ClC}_{6} \mathrm{H}_{4}$ & $\mathrm{Ph}$ & $\mathrm{Ph}$ & 86 \\
\hline 6 & $6 \mathbf{e}$ & 3-Thenyl & $\mathrm{Ph}$ & $2-\mathrm{ClC}_{6} \mathrm{H}_{4}$ & 76 \\
\hline 7 & $6 f$ & 3-Thenyl & $\mathrm{Ph}$ & $4-\mathrm{FC}_{6} \mathrm{H}_{4}$ & 87 \\
\hline 8 & $6 \mathrm{~g}$ & 3-Thenyl & $\mathrm{Ph}$ & $2,4-\mathrm{Cl}_{2} \mathrm{C}_{6} \mathrm{H}_{3}$ & 92 \\
\hline 9 & $6 \mathrm{~h}$ & $n$-Hexyl & $\mathrm{Ph}$ & $4-\mathrm{CH}_{3} \mathrm{OC}_{6} \mathrm{H}_{4}$ & 73 \\
\hline 10 & $6 \mathbf{i}$ & $n$-Hexyl & $\mathrm{Ph}$ & $3,4,5-\left(\mathrm{CH}_{3} \mathrm{O}\right)_{3} \mathrm{C}_{6} \mathrm{H}_{2}$ & 79 \\
\hline 11 & $\mathbf{6 j}$ & $n$-Hexyl & $\mathrm{Ph}$ & 2-Thenyl & 74 \\
\hline 12 & $6 \mathbf{k}$ & $n$-Hexyl & $\mathrm{Ph}$ & $2-\mathrm{ClC}_{6} \mathrm{H}_{4}$ & 66 \\
\hline 13 & 61 & $s$-Butyl & $\mathrm{Ph}$ & $4-\mathrm{CH}_{3} \mathrm{C}_{6} \mathrm{H}_{5}$ & 54 \\
\hline 14 & $6 \mathrm{~m}$ & $s$-Butyl & $\mathrm{Ph}$ & $4-\mathrm{CH}_{3} \mathrm{OC}_{6} \mathrm{H}_{5}$ & 43 \\
\hline 15 & 6n & 1-naphthyl & $\mathrm{Ph}$ & 4- $\mathrm{CH}_{3} \mathrm{C}_{6} \mathrm{H}_{5}$ & 73 \\
\hline 16 & & ph & $\mathrm{Ph}$ & $3-\mathrm{HO}-4-\mathrm{CH}_{3} \mathrm{OC}_{6} \mathrm{H}_{3}$ & $\mathrm{NR}^{c}$ \\
\hline 17 & & $n$-Hexyl & $\mathrm{Ph}$ & $4-\mathrm{HOC}_{6} \mathrm{H}_{4}$ & $\mathrm{NR}^{c}$ \\
\hline
\end{tabular}

${ }^{a}$ Unless otherwise noted, the reaction was performed by employing organozinc halide $(4.5 \mathrm{mmol}), \mathrm{N}, \alpha$-diphenyl nitrone $(3 \mathrm{mmol})$ in THF $(20 \mathrm{~mL}) .{ }^{b}$ Isolated yield. ${ }^{c} \mathrm{NR}=$ No reaction.

The $O$-trimethylsilyl hydroxylamine ethers can be easily 15 converted into corresponding amines by simply stirring the ethers with a zinc-copper couple in a saturated aqueous $\mathrm{NH}_{4} \mathrm{Cl}$ at $50{ }^{\circ} \mathrm{C}$ for 1 hour. As illustrated in Scheme 1, the substrates $\mathbf{4 a}$ and $\mathbf{4 f}$ were reduced using this protocol to the corresponding amines in $77 \%$ and $72 \%$ isolated yields respectively.<smiles>[R][C-](C)ON(c1ccccc1)C(Cc1ccccc1)c1ccccc1</smiles>

4a: $\mathrm{R}=4-\mathrm{OCH}_{3}$

20

Scheme 1. Reduction of $O$-TMS hydroxylamine ethers to amines

To test the application of our TMSCl promoted organozinc protocol for natural product synthesis, we prepared a cyclic nitrone 9 from D-mannose ${ }^{21}$ (Scheme 2). Gratifyingly, 9 reacted 25 with phenylzinc bromide, [no reaction without $\mathrm{TMSCl}$ ] in the presence of two equivalents of $\mathrm{TMSCl}$ at room temperature to furnish the desired $O$-TMS-hydroxylamine ether $\mathbf{1 0}$ in $84 \%$ yield stereospecifically. Upon further stirring the reaction mixture at room temperature for 2 hours in saturated aqueous $\mathrm{NH}_{4} \mathrm{Cl}$ 30 solution and with zinc metal in the presence of catalytic amount of $\mathrm{Cu}(\mathrm{OAc})_{2}, \mathbf{1 0}$ was completely reduced to the pyrrolidine $\mathbf{1 1}$ in $91 \%$ yield. Deprotection of $\mathbf{1 1}$ by catalytic hydrogenation in acid solution gave the $\alpha$-ethyl polyhydroxylated pyrrolidine $\mathbf{1 2}$ in quantative yield which is a structural analogue of 2,5-

35 dideoxy-2,5-imino-D-glycero-D-manno-heptitol (homo DMDP), a natural selective inhibitors of $\beta$-glucosidases. ${ }^{3 \mathrm{~b}}$

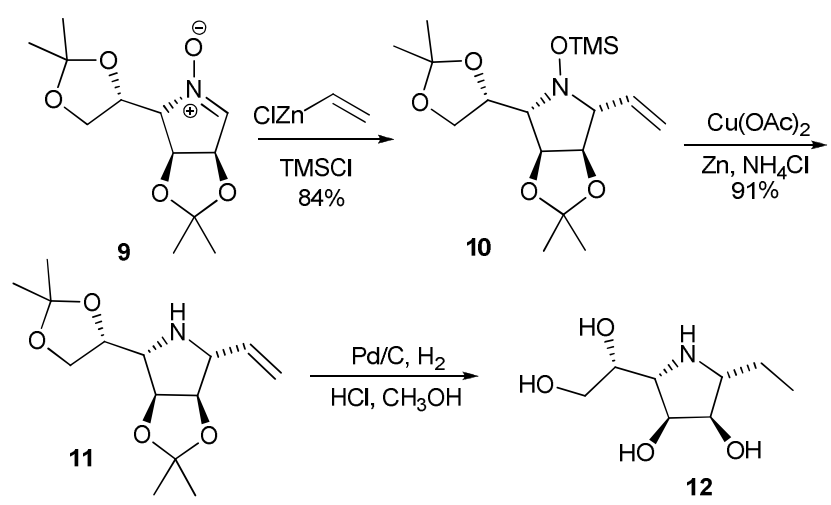

Scheme 2. Synthesis of branched polyhydroxylated pyrrolidine $\mathbf{1 2}$

In conclusion, we have successfully developed a new method 40 for the synthesis of substituted $O$-trimethylsilyl hydroxylamine ethers via a TMSCl-catalyzed nucleophilic addition of organozinc halides to nitrones. To the best of our knowledge, the present reaction is the first example of a TMSCl catalyzed nucleophilic addition reaction of organozinc reagents with nitrones whereby 45 the TMSCl acts both as a reaction promoter and a good protection reagent for the sensitive $N$-hydroxylamine group. Furthermore, the $O$-TMS protected hydroxylamine ethers can be easily reduced by zinc to the corresponding amines. This protocol offers a practically useful method in natural products synthesis.

\section{${ }_{50}$ Notes and references}

${ }^{a}$ College of Chemistry and Chemical Engineering, Northwest Normal University, Lanzhou, An'ning East Road No.967, Gansu Province, 730070, P. R. China. E-mail: fuying@iccas.ac.cn.

${ }^{b}$ Health Innovations Research Institute \& School of Applied Sciences, RMIT 55 University, Melbourne, 3001 Australia.

$\dagger$ Electronic Supplementary Information (ESI) available: [details of any supplementary information available should be included here]. See DOI: $10.1039 / \mathrm{b} 000000 \mathrm{x} /$

\$ Footnotes should appear here. These might include comments relevant 60 to but not central to the matter under discussion, limited experimental and spectral data, and crystallographic data.

1 A. G. Dyker, K. R. Lees, Stroke, 1999, 30, 1796-1801.

2 A. K. Desai, G. T. Grossberg. Expert Rev Neurother. 2005, 5, 563580 .

653 (a) A. Welter, J. Jadot, G. Dardenne, M. Marlier, J. Casimir, Phytochemistry, 1976, 15, 747-749; (b) T. M. Wrodnigg, Monatsh. Chem. 2002, 133, 393-426.

4 (a) A. Hamze, B. Tréguier, J.-D. Brion, M. Alami, Org. Biomol. Chem., 2011, 9, 6200-6204; (b) E. L. Gall, C. Haurena, S. Sengmany,

70 T. Martens, M. Troupel, J. Org. Chem., 2009, 74, 7970-7973.

5 For reviews on nucleophilic addition of organometallics to nitrones, see: (a) R. Bloch, Chem. Rev. 1998, 98, 1407-1438; (b) P. Merino, S. Franco, F. L. Merchan, T. Tejero, Synlett 2000, 442-454; (c) M. Lombardo, C. Trombini, Curr. Org. Chem. 2002, 6, 695-713; (d) P. 75 Merino, C. R. Chimie 2005, 8, 775-788; (e) M. Lombardo, C. Trombini, Synthesis 2000, 759-774.

6 (a) S. K.Patel, K. Murat, S. Py, Y. Vallée, Org. Lett. 2003, 5, 40814084; (b) M. Bonanni, M. Marradi, S. Cicchi, C. Faggi, A. Goti, Org. Lett. 2005, 7, 319-322; (c) Y. Kazuta, H. Abe, A. Matsuda, S. Shuto, $80 \quad$ J. Org. Chem. 2004, 69, 9143-9150. 
7 (a) P. Merino, I. Delso, V. Mannucci, T. Tejero, Tetrahedron Lett, 2006, 47, 3311-3314; (b) A. Pernet-Poil-Chevrier, F. Cantagrel, K. Le Jeune, C. Philouze, P. Y. Chavant, Tetrahedron: Asymmetry 2006, 17, 1969-1974; (c) M. Chrzanowska, M. D. Rozwadowska, Chem.

5 Rev. 2004, 104, 3341-3370; (d) P. Merino, V. Mannucci, T. Tejero, Eur. J. Org. Chem. 2008, 3943-3959.

8 (a) W. Zhang, K. Sato, A. Kato, Y.-M. Jia, X.-G. Hu, Wilson, F. X. Well, R. van Well, G. Horne, G. W. J. Fleet, R. J. Nash, C.-Y. Yu, Org. Lett. 2011, 13, 4414-4417; (b) O. P. Bande, V. H. Jadhav, V. G. 10 Puranik, D. D. Dhavale, M. Lombardo. Tetrahedron Lett. 2009, 50, 6906-6908; (c) X.-G. Hu, B. Bartholomew, R. J. Nash, F. X. Wilson, G. W. J. Fleet, S. Nakagawa, A. Kato, Y.-M. Jia, R. van Well, C. Y. Yu, Org. Lett. 2010, 12, 2562-2565; (d) J. A. Tamayo, F. Franco, D. Lo Re, F. Sánchez-Cantalejo, J. Org. Chem., 2009, 74, 5679-5682.

159 For reviews on organozinc chemistry, see: (a) Z. Rappoport, I.Marek, The chemistry of organozinc Compounds: R-Zn, Wiley, Chichester, UK, 2006; (b) P. Knochel, P. Jones, Organozinc Reagents: A Practical Approach. Oxford University Press, Oxford, 1999; (c) E. Erdik, Organozinc Reagents in Organic Synthesis. CRC: New York, 20 1996; (d) P. Knochel, R. D. Singer, Chem. Rev. 1993, 93, 2117-2188.

10 Y. Ukaji, Y. Kenmoku, K. Inomata, Tetrahedron: Asymmetry 1996, 7, 53-56.

11 (a) S. Pinet, S. U. Pandya, P. Y. Chavant, A. Ayling, Y. Vallee, Org. Lett., 2002, 4, 1463-1466; (b) F. Cantagrel, S. Pinet, Y. Gimbert, P. Y.

25 Chavant, Eur. J. Org. Chem. 2005, 2694-2701; (c) W. Wei, M. Kobayashi, Y. Ukaji, K. Inomata, Chem. Lett. 2006, 35, 176-177; (d) L. Zani, S. Alesi, P. G. Cozzi, C. Bolm, J. Org. Chem. 2006, 71, 1558-1562.

12 A. Fiumana, M. Lombardo, C. Trombini, J. Org. Chem. 1997, 62, 5623-5626.

13 (a) S. Wang, C. T. Seto, Org. Lett., 2006, 8, 3979-3982; (b) N. PraveenGanesh, C. de Candia, A. Memboeuf, G. Lendvay, Y. Gimbert, P.Y. Chavant, J. Organomet. Chem. 2010, 695, 2447-2454; (c) C. Eriksson, K. Sjödin, F. Schlyter, H. E. Högberg, Tetrahedron:

35 Asymm., 2006, 17, 1074-1080; (d) S. U. Pandya, S. Pinet, P. Y. Chavant, Y. Vallée, Eur. J. Org. Chem. 2003, 19, 3621-3627.

14 (a) Y. Ukaji, K. Inomata, Synlett 2003, 1075-1087; (b) Y. Ukaji, Y. Yoshida, K. Inomata, Tetrahedron: Asymmetry 2000, 11, 733-736.

15 P. Knochel, H. Leuser, L.-Z. Gong, S. Perrone, F. F. Kneisel, In

40 Handbook of Functionalized Organometallics: Applications in Synthesis, P. Knochel, Ed.; Wiley-VCH: Weinheim, Germany, 2005; Vol. 1. 251.

16 (a) J.-X. Wang, Y. Fu, Y. L. Hu, Angew. Chem. 2002, 114, 2881-2884; Angew. Chem. Int. Ed. 2002, 41, 2757-2760; (b) J.-X. Wang, Y. Fu,

45 Y. L. Hu, Synthesis, 2003, 1506-1510; (c) Y. Fu, X. Hu, Y. Chen, Y. Yang, H. Hou, Y. Hu, synthesis, 2012, 44, 1030-1036; (d) J.-X. Wang, K. Wang, L. Zhao, H. Li, Y. Fu, Y. Hu, Adv. Synth. Catal. 2006, 348, 1262-1270.

17 (a) Y. Fu, J.-X. Wang, K. Wang, Y. Hu, Tetrahedron 2008, 64, 11124-11128; (b) Y. Hu, J.-X. Wang, W. Li, Chem lett. 2001, 174175.

18 (a) C. Palomo, M. Oiarbide, E. Arceo, J. M. García, R. López, A. González, A. Linden, Angew. Chem. 2005, 117, 6343-6346; Angew. Chem. Int. Ed. 2005, 44, 6187-6190; (b) T. Kano, T. Hashimoto, K.

55 Maruoka, J. Am. Chem. Soc., 2005, 127, 11926-11927.

19 H. P. Knoess, M. T. Furlong, M. J. Rozema, P. Knochel, J. Org. Chem. 1991, 56, 5974-5978.

20 Y. Kazuta, S. Shuto, A. Matsuda, Tetrahedron Lett. 2000, 41, $5373-$ 5377.

6021 Nitrone 9 was prepared by similar method as S. Desvergnes, S. Py, Y. Vallée, J. Org. Chem. 2005, 70, 1459-1462. 


\section{Supporting Information}

\section{Trimethylsilyl Chloride Promoted Synthesis of $\alpha$-branched amines by Nucleophilic Addition of Organozinc Halides to Nitrones}

Ying Fu, ${ }^{* a}$ Yanhua Liu, ${ }^{a}$ Yaojuan Chen, ${ }^{a}$ Helmut M. Hügel, ${ }^{b}$ Danfeng Huang ${ }^{a}$ and Yulai Hu ${ }^{a}$

[a]. College of Chemistry and Chemical Engineering, Northwest Normal University, Lanzhou, Gansu 730070, P. R. China.

[b]. Health Innovations Research Institute \& School of Applied Sciences, RMIT University Melbourne, 3001 Australia.

fuying@iccas.ac.cn

\section{Table of Contents}

Table of Contents

S1

General Experimental Section

S2

General Procedure A: TMSCl promoted reaction of organozinc halides with

nitrones

S2

Synthesis and characterization of products 4 and 6:

S3-S20

General procedure B: Reduction of O-trimethylsilyl hydroxylamines into

S20

amine.

S24

Reference

S25-S99

NMR Spectra of Products 


\section{General Experimental Section:}

Analytic methods. All the reactions were carried out under argon atmosphere using standard Schlenk technique. ${ }^{1} \mathrm{H}$ NMR (400 MHz), and ${ }^{13} \mathrm{C}$ NMR (100 MHz) were recorded on Bruker AV400 NMR spectrometer with $\mathrm{CDCl}_{3}$ as solvent. Chemical shifts of ${ }^{1} \mathrm{H}$ and ${ }^{13} \mathrm{C}$ NMR spectra are reported in parts per million (ppm) with TMS as an internal standard. All coupling constants ( $\mathrm{J}$ values) were reported in Hertz (Hz). Multiplicities are reported as follows: singlet (s), doublet (d), doublet of doublets (dd), doublet of doublet of doublets (ddd), doublet of triplets (dt), triplet (t), triplet of doublets (td), quartet (q), and multiplet (m). Column chromatography was performed on silica gel $300 \square 400$ mesh. Analytical thin $\square$ layer chromatography (TLC) was performed on pre-coated, glass $\square$ backed silica gel plates. Visualization of the developed chromatogram was performed by UV absorbance (254 $\mathrm{nm}$ ). IR spectra were recorded as $\mathrm{KBr}$ disks on a Alpha Centauri FT-IR spectrometer. EI mass spectra and HRMS were done on QP-1000A GCMS spectrometer by EI ionization at $70 \mathrm{eV}$. ESI mass spectra were recorded on a Bruker Esquire 6000.

General preparation for chemicals. The substrates N-phenylhydroxylamine ${ }^{[1]}$ and N-benzyl hydroxlamine ${ }^{[2]}$ was prepared according to the known procedure. $\alpha, \mathrm{N}$-diphenyl nitrone derivatives were prepared from benzaldehydes and N-hydroxylamines following a modified literature procedure. ${ }^{[3]}$ Benzylic zinc halides were prepared following the Knochel's procedure. ${ }^{[4]}$ Arylic zinc halides and alkyl were prepared by transmetallation of corresponding Grignard reagents with $\mathrm{ZnCl}_{2}$ and were titrationed by standard procedure. ${ }^{[5]}$ All other reagents used are from commercial sources and used without any further purification.

\section{General Procedure A: TMSCl promoted reaction of organozinc halides with nitrones}

General procedure for the TMSCl promoted reaction of organozinc halides with nitrones. The organozinc halides were used immediately after preparation. TMSCl $(0.65 \mathrm{~g}, 0.78 \mathrm{ml}, 6.0 \mathrm{mmol})$ in THF ( $5 \mathrm{~mL})$ was slowly added to a solution of organozinc halides $(4.5 \mathrm{mmol})$ and nitrone $(3.0 \mathrm{mmol})$ in $\mathrm{THF}(10 \mathrm{ml})$ at room temperature. The reaction mixture was heated to $30{ }^{\circ} \mathrm{C}$ and stirred at this temperature for $4 \mathrm{~h}$. After cooling to room temperature, the reaction solution was quenched with saturated $\mathrm{NH}_{4} \mathrm{Cl}(10 \mathrm{~mL}), 20 \mathrm{~mL}$ of ethyl acetate was then added and the organic phase was separated, washed with $10 \mathrm{~mL}$ of water and then with $10 \mathrm{ml}$ of brine. The water phase was extracted with ethyl acetate $(2 \times 10 \mathrm{~mL})$. The combined organic phase was then dried $\left(\mathrm{Na}_{2} \mathrm{SO}_{4}\right)$ and concentrated under reduced pressure. Column chromatography on silica gel with petroleum/ethyl acetate as eluent gave the protected O-trimethylsilyl hydroxylamine ethers. 


\section{Synthesis and characterization of products 4 and 6:}

4a: N-(1-(4-methoxyphenyl)-2-phenylethyl)-N-phenyl-O-(trimethylsilyl)hydroxylamine

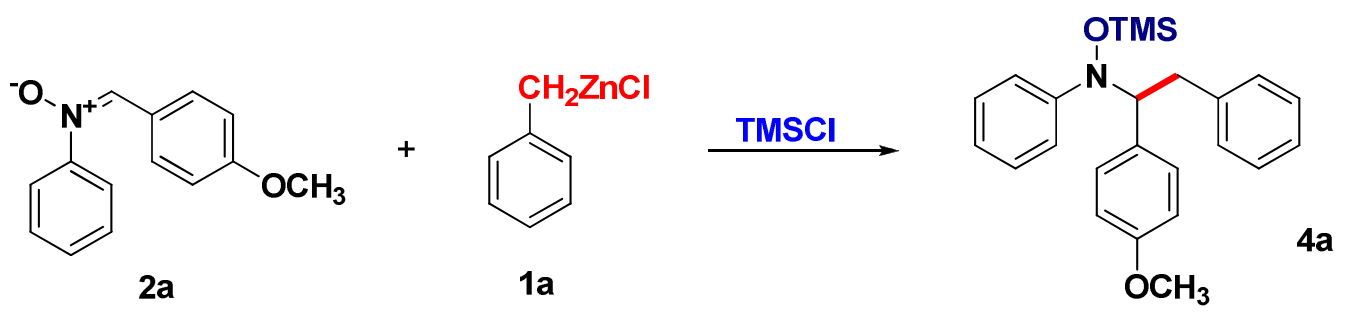

According to general procedure A: A solution of TMSCl $(0.65 \mathrm{~g}, 0.78 \mathrm{ml}, 6.0 \mathrm{mmol})$ in THF $(5 \mathrm{~mL})$ was added slowly to a mixture of N-phenyl-C-(4-methoxyphenyl) nitrone $\mathbf{2 a}(0.68 \mathrm{~g}, 3.0 \mathrm{mmol})$, benzylzinc chloride $(4.5 \mathrm{mmol})$ in $10 \mathrm{ml}$ of THF under room temperature. The reaction mixture was stirred at this temperature for half an hour. The pure product was obtained as colorless oil (0.94 g, 2.4 mmol, $80 \%)$ after purification on silica gel (10:1 petroleum ether : EtOAc).

IR $(\mathrm{KBr}) \vee\left(\mathrm{cm}^{-1}\right): 3424,3062,3028,2958,2836,1609,1487,1453,1249,1207,919 ;{ }^{1} \mathrm{H}$ NMR (400 $\left.\mathrm{MHz}, \mathrm{CDCl}_{3}\right) \delta(\mathrm{ppm}):-0.05(\mathrm{~s}, 9 \mathrm{H}), 3.42(\mathrm{dd}, J=13.6,10.7 \mathrm{~Hz}, 1 \mathrm{H}), 3.53(\mathrm{dd}, J=13.8,3.9 \mathrm{~Hz}, 1 \mathrm{H})$, $3.73(\mathrm{~s}, 3 \mathrm{H}), 4.44(\mathrm{dd}, J=10.6,3.9 \mathrm{~Hz}, 1 \mathrm{H}), 6.70(\mathrm{~d}, J=8.4 \mathrm{~Hz}, 2 \mathrm{H}), 7.17(\mathrm{dd}, J=7.4,0.9 \mathrm{~Hz}, 3 \mathrm{H})$, $7.24(\mathrm{~d}, J=6.2 \mathrm{~Hz}, 1 \mathrm{H}), 7.29(\mathrm{t}, J=13.7 \mathrm{~Hz}, 6 \mathrm{H}), 7.40(\mathrm{dd}, J=10.8,2.9 \mathrm{~Hz}, 2 \mathrm{H}) ;{ }^{13} \mathrm{C}$ NMR $(100$ $\left.\mathrm{MHz}, \mathrm{CDCl}_{3}\right) \delta(\mathrm{ppm}):-0.5,36.1,55.0,75.2,112.8,120.7,123.3,125.6,128.0,128.0,129.2,131.1$, 139.4, 152.6, 158.7. MS (EI, $70 \mathrm{eV}): \mathrm{m} / \mathrm{z}(\%)=211(100), 391\left(0.06,[\mathrm{M}]^{+}\right)$; Anal. Calcd for $\mathrm{C}_{24} \mathrm{H}_{29} \mathrm{NO}_{2} \mathrm{Si}: \mathrm{C}, 73.61 ; \mathrm{H}, 7.46 ; \mathrm{N}, 3.58$. Found: C, 73.38; H, 6.96; N, 3.43.

\section{4b: N-(1,2-diphenylethyl)-N-phenyl-O-(trimethylsilyl)hydroxylamine}<smiles>[O-][N+](=Cc1ccccc1)c1ccccc1</smiles>

2b<smiles>Cl[Te]Cc1ccccc1</smiles>

$1 a$

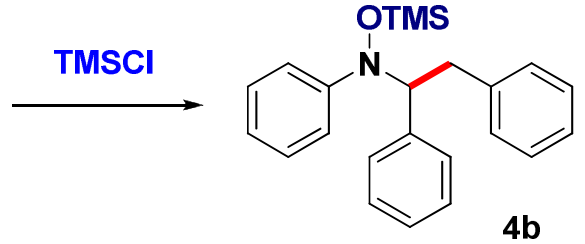

According to general procedure A: A solution of TMSCl $(0.65 \mathrm{~g}, 0.78 \mathrm{ml}, 6.0 \mathrm{mmol})$ in THF (5 mL) was slowly added to a solution of $\mathrm{N}$, $\alpha$-diphenyl nitrone $\mathbf{2 b}(0.60 \mathrm{~g}, 3.0 \mathrm{mmol})$ and benzylzinc chloride $(4.5 \mathrm{mmol})$ in $10 \mathrm{ml}$ of $\mathrm{THF}$ at room temperature. The reaction mixture was stirred at this temperature for half an hour. The pure product was obtained as colorless oil $(0.90 \mathrm{~g}, 4.2 \mathrm{mmol}, 83 \%)$ after purification on silica gel (20:1 petroleum ether : EtOAc).

IR $(\mathrm{KBr}) \vee\left(\mathrm{cm}^{-1}\right): 3063,3029,2959,1595,1487,1452,1205,878,843 ;{ }^{1} \mathrm{H}$ NMR $\left(400 \mathrm{MHz}, \mathrm{CDCl}_{3}\right) \delta$ (ppm): -0.07 (s, 9H), 3.23-3.35 (m, 2H), 4.48 (q, $J=4.0 \mathrm{~Hz}, 1 \mathrm{H}), 6.93-7.02(\mathrm{~m}, 3 \mathrm{H}), 7.11(\mathrm{dt}, J=7.2$, $1.6 \mathrm{~Hz}, 1 \mathrm{H}), 7.12-7.21(\mathrm{~m}, 4 \mathrm{H}), 7.21-7.32(\mathrm{~m}, 7 \mathrm{H}) ;{ }^{13} \mathrm{C} \mathrm{NMR}\left(100 \mathrm{MHz}, \mathrm{CDCl}_{3}\right) \delta$ (ppm): -0.5, 35.9, 
75.8, 120.7, 123.5, 125.6, 127.2 127.5, 128.0, 128.1, 129.2, 130.1, 138.3, 139.3, 152.6. MS (EI, 70 eV): $\mathrm{m} / \mathrm{z}(\%)=270(100), 361\left(1.0,[\mathrm{M}]^{+}\right)$. Anal. Calcd for $\mathrm{C}_{23} \mathrm{H}_{27} \mathrm{NOSi}$ : C, 76.41; H, 7.53; N, 3.87. Found: C, 76.11; H, 7.22; N, 3.58.

4c: N-(1-(2-chlorophenyl)-2-phenylethyl)-N-phenyl-O-(trimethylsilyl)hydroxylamine<smiles>CON(c1ccccc1)C(Cc1ccccc1)c1ccccc1Cl</smiles>

According to general procedure A: A solution of TMSCl $(0.65 \mathrm{~g}, 0.78 \mathrm{ml}, 6.0 \mathrm{mmol})$ in THF $(5 \mathrm{~mL})$ was added slowly to a mixture of N-phenyl-C-(2-chlorophenyl) nitrone 2c (0.70 g, $3.0 \mathrm{mmol})$, benzylzinc chloride $(4.5 \mathrm{mmol})$ in $10 \mathrm{ml}$ of THF under room temperature. The reaction mixture was stirred at this temperature for half an hour. The pure product was obtained as colorless oil ( $0.66 \mathrm{~g}, 1.7$ mmol, $56 \%)$ after purification on silica gel (30:1 petroleum ether : EtOAc).

IR $(\mathrm{KBr}) \vee\left(\mathrm{cm}^{-1}\right): 3339,3063,3027,1596,1487,1449,1088,1034,921,880 ;{ }^{1} \mathrm{H}$ NMR (400 MHz, $\left.\mathrm{CDCl}_{3}\right) \delta(\mathrm{ppm}):-0.29(\mathrm{~s}, 9 \mathrm{H}), 3.12(\mathrm{dd}, J=14.6,3.8 \mathrm{~Hz}, 1 \mathrm{H}), 3.32(\mathrm{dd}, J=14.3,11.4 \mathrm{~Hz}, 1 \mathrm{H}), 5.18$ $(\mathrm{dd}, J=11.2,3.6 \mathrm{~Hz}, 1 \mathrm{H}), 6.86(\mathrm{~d}, J=6.8 \mathrm{~Hz}, 2 \mathrm{H}), 6.94(\mathrm{tq}, J=7.2,1.6 \mathrm{~Hz}, 2 \mathrm{H}), 6.98-7.08(\mathrm{~m}, 3 \mathrm{H})$, $7.14(\mathrm{td}, J=7.6,1.2 \mathrm{~Hz}, 1 \mathrm{H}), 7.16-7.28(\mathrm{~m}, 5 \mathrm{H}), 7.56(\mathrm{dd}, J=7.9,1.7 \mathrm{~Hz}, 1 \mathrm{H}) ;{ }^{13} \mathrm{C}$ NMR $(100 \mathrm{MHz}$, $\left.\mathrm{CDCl}_{3}\right) \delta(\mathrm{ppm}):-0.9,32.6,70.0,119.6,123.2,125.7,125.8,128.0,128.2,128.6,128.9,131.0,135.9$, 136.1, 138.5, 152.7. MS (EI, $70 \mathrm{eV}): \mathrm{m} / \mathrm{z}(\%)=304(100), 395\left(1.9,[\mathrm{M}]^{+}\right)$. Anal. Calcd for $\mathrm{C}_{23} \mathrm{H}_{26}$ CINOSi: C, 69.76; H, 6.62; N, 3.54. Found: C, 70.04; H, 6.36; N, 3.33.

\section{4d: N-(1-(4-chlorophenyl)-2-phenylethyl)-N-phenyl-O-(trimethylsilyl)hydroxylamine}

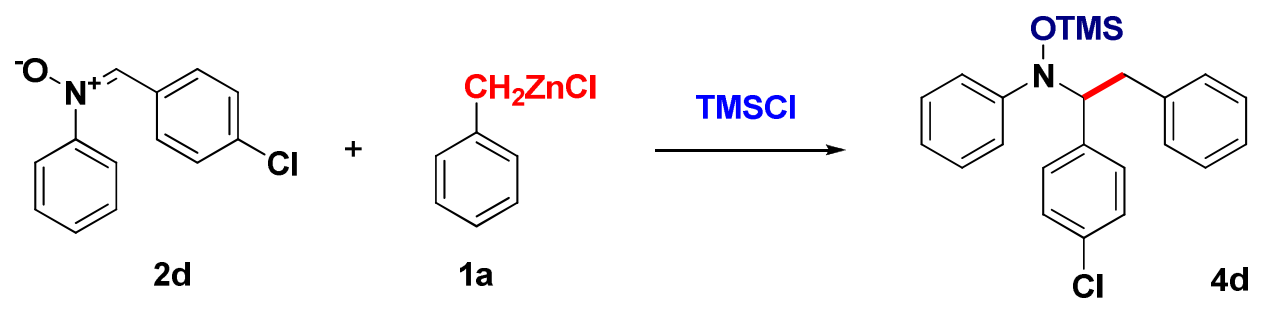

According to general procedure A: A solution of TMSCl $(0.65 \mathrm{~g}, 0.78 \mathrm{ml}, 6.0 \mathrm{mmol})$ in THF $(5 \mathrm{~mL})$ was added slowly to a mixture of N-phenyl-C-(4-chlorophenyl) nitrone $\mathbf{2 d}(0.70 \mathrm{~g}, 3.0 \mathrm{mmol})$,

benzylzinc chloride $(4.5 \mathrm{mmol})$ in $10 \mathrm{ml}$ of THF under room temperature. The reaction mixture was stirred at this temperature for half an hour. The pure product was obtained as colorless oil $(0.97 \mathrm{~g}, 2.46$ mmol, $82 \%)$ after purification on silica gel (30:1 petroleum ether : EtOAc). 
IR $(\mathrm{KBr}) \vee\left(\mathrm{cm}^{-1}\right): 3028,2960,1596,1490,1250,880,844 ;{ }^{1} \mathrm{H} \mathrm{NMR}\left(400 \mathrm{MHz}, \mathrm{CDCl}_{3}\right) \delta(\mathrm{ppm}): 0.02$ (s, 9H), $3.26(\mathrm{dd}, J=13.6,10.8 \mathrm{~Hz}, 1 \mathrm{H}), 3.40(\mathrm{dd}, J=13.6,4.0 \mathrm{~Hz}, 1 \mathrm{H}), 4.49$ (q, $J=4.0 \mathrm{~Hz}, 1 \mathrm{H}), 6.93-$ $7.20(\mathrm{~m}, 12 \mathrm{H}), 7.20-7.26(\mathrm{~m}, 2 \mathrm{H}) ;{ }^{13} \mathrm{C} \mathrm{NMR}\left(100 \mathrm{MHz}, \mathrm{CDCl}_{3}\right) \delta(\mathrm{ppm}):-0.5,36.7,75.3,120.8,123.8$, 125.9, 127.6, 128.1, 129.1, 131.3, 133.0, 136.6, 138.8, 152.4; MS (EI, 70 eV): m/z (\%) = 304 (100), $395\left(4.5,[\mathrm{M}]^{+}\right)$. Anal. Calcd for $\mathrm{C}_{23} \mathrm{H}_{26} \mathrm{ClNOSi}$ : C, 69.76; H, 6.62; N, 3.54. Found: C, 69.93; H, 6.42; $\mathrm{N}, 3.75$.

4e: N-(1-(2,4-dichlorophenyl)-2-phenylethyl)-N-phenyl-O-(trimethylsilyl)hydroxylamine

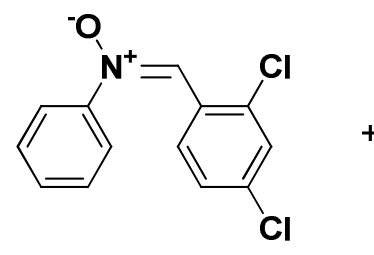

$2 e$<smiles>Cl[Te]Cl</smiles>

$1 a$

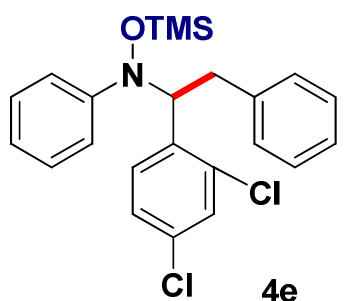

$4 e$

According to general procedure A: A solution of TMSCl $(0.65 \mathrm{~g}, 0.78 \mathrm{ml}, 6.0 \mathrm{mmol})$ in THF $(5 \mathrm{~mL})$ was added slowly to a mixture of N-phenyl-C-(2,4-dichlorophenyl) nitrone $2 \mathrm{e}(0.80 \mathrm{~g}, 3.0 \mathrm{mmol})$, benzylzinc chloride $(4.5 \mathrm{mmol})$ in $10 \mathrm{ml}$ of THF under room temperature. The reaction mixture was stirred at this temperature for half an hour. The pure product was obtained as colorless oil (0.92 $\mathrm{g}, 2.13$ mmol, $71 \%)$ after purification on silica gel (30:1 petroleum ether : EtOAc).

IR $(\mathrm{KBr}) \vee\left(\mathrm{cm}^{-1}\right): 3029,2959,1593,1488,1451,1250,880,843,738,698 ;{ }^{1} \mathrm{H}$ NMR (400 MHz, $\left.\mathrm{CDCl}_{3}\right) \delta(\mathrm{ppm}):-0.01(\mathrm{~s}, 9 \mathrm{H}), 3.36(\mathrm{dd}, J=14.4,3.6 \mathrm{~Hz}, 1 \mathrm{H}), 3.49(\mathrm{dd}, J=14.4,11.2 \mathrm{~Hz}, 1 \mathrm{H}), 5.36$ (ddd, $J=11.2,4.0,1.6 \mathrm{~Hz}, 1 \mathrm{H}), 7.10(\mathrm{~d}, J=8.0 \mathrm{~Hz}, 2 \mathrm{H}), 7.15-7.30(\mathrm{~m}, 4 \mathrm{H}), 7.36$ (ddd, $J=8.4,3.2,1.6$ $\mathrm{Hz}, 1 \mathrm{H}), 7.40-7.50(\mathrm{~m}, 5 \mathrm{H}), 7.74(\mathrm{dd}, J=8.4,1.5 \mathrm{~Hz}, 1 \mathrm{H}) ;{ }^{13} \mathrm{C} \mathrm{NMR}\left(100 \mathrm{MHz}, \mathrm{CDCl}_{3}\right) \delta(\mathrm{ppm}):-0.9$, 33.9, 69.4, 109.7, 110.1, 120.0, 123.5, 125.9, 128.1, 128.2, 128.9, 139.0, 141.5, 141.5, 152.1, 152.4. MS (EI, $70 \mathrm{eV}): \mathrm{m} / \mathrm{z}(\%)=338(100), 429\left(1.8,[\mathrm{M}]^{+}\right)$; Anal. Calcd for $\mathrm{C}_{23} \mathrm{H}_{25} \mathrm{Cl}_{2} \mathrm{NOSi}$ : C, 64.18; $\mathrm{H}$, 5.85; N, 3.25. Found: C, 63.87; H, 5.52; N, 3.07.

\section{4f: N-phenyl-N-(2-phenyl-1-(3,4,5-trimethoxyphenyl)ethyl)-O-(trimethylsilyl)hydroxylamine}
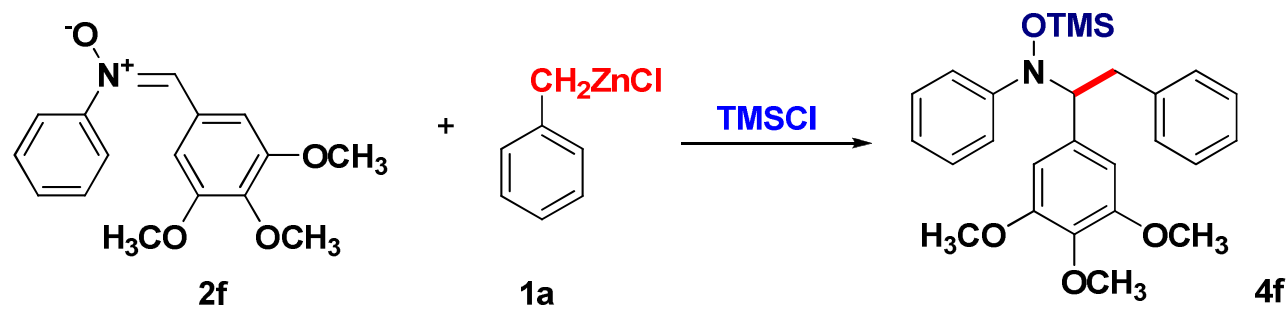

According to general procedure A: A solution of $\mathrm{TMSCl}(0.65 \mathrm{~g}, 0.78 \mathrm{ml}, 6.0 \mathrm{mmol})$ in $\mathrm{THF}(5 \mathrm{~mL})$ was added slowly to a mixture of N-phenyl-C-(3,4,5-trimethoxyphenyl) nitrone $2 \mathbf{f}(0.86 \mathrm{~g}, 3.0 \mathrm{mmol})$, benzylzinc chloride $(4.5 \mathrm{mmol})$ in $10 \mathrm{ml}$ of THF under room temperature. The reaction mixture was 
stirred at this temperature for half an hour. The pure product was obtained as colorless oil (1.02 $\mathrm{g}, 2.25$ mmol, 75\%) after purification on silica gel (10:1 petroleum ether : EtOAc).

IR $(\mathrm{KBr}) \vee\left(\mathrm{cm}^{-1}\right): 2953,2838,1503,1457,1422,1347,1244,1126,1101,922,880,844 ;{ }^{1} \mathrm{H}$ NMR $\left(400 \mathrm{MHz}, \mathrm{CDCl}_{3}\right) \delta(\mathrm{ppm}):-0.13(\mathrm{~s}, 9 \mathrm{H}), 3.11(\mathrm{dd}, J=13.7,10.5 \mathrm{~Hz}, 1 \mathrm{H}), 3.20(\mathrm{dd}, J=13.9,3.9 \mathrm{~Hz}$, $1 \mathrm{H}), 3.59$ (s, 6H), $3.68(\mathrm{~s}, 3 \mathrm{H}), 4.30(\mathrm{q}, J=3.9 \mathrm{~Hz}, 1 \mathrm{H}), 6.31(\mathrm{~s}, 2 \mathrm{H}), 6.84-6.96(\mathrm{~m}, 4 \mathrm{H}), 6.96-7.06(\mathrm{~m}$, $4 \mathrm{H}), 7.17(\mathrm{t}, J=8.4 \mathrm{~Hz}, 2 \mathrm{H}) ;{ }^{13} \mathrm{C}$ NMR $\left(100 \mathrm{MHz}, \mathrm{CDCl}_{3}\right) \delta(\mathrm{ppm}):-0.7,36.0,55.7,60.5,75.7,107.0$, $120.4,123.3,125.6,127.8,127.9,128.9,130.4,137.1,138.9,152.0,152.2$. HRMS (ESI): calcd. for $\mathrm{C}_{26} \mathrm{H}_{34} \mathrm{NO}_{4} \mathrm{Si}[\mathrm{M}]^{+}:$452.2257, found: 452.2258 .

4g: 2-methoxy-5-(2-phenyl-1-(phenyl((trimethylsilyl)oxy)amino)ethyl)phenol

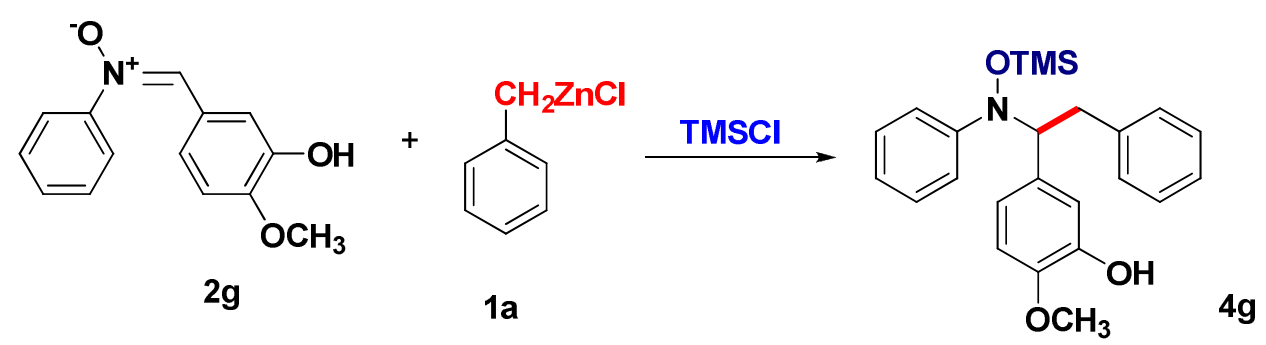

According to general procedure A: A solution of TMSCl $(0.65 \mathrm{~g}, 0.78 \mathrm{ml}, 6.0 \mathrm{mmol})$ in THF $(5 \mathrm{~mL})$ was added slowly to a mixture of N-phenyl-C-(3-hydroxy-4-methoxyphenyl) nitrone $2 \mathrm{~g}(0.73 \mathrm{~g}, 3.0$ mmol), benzylzinc chloride $(4.5 \mathrm{mmol})$ in $10 \mathrm{ml}$ of THF under room temperature. The reaction mixture was stirred at this temperature for half an hour. The pure product was obtained as colorless oil $(0.80 \mathrm{~g}$, $1.98 \mathrm{mmol}, 66 \%)$ after purification on silica gel (10:1 petroleum ether : EtOAc).

IR $(\mathrm{KBr}) \vee\left(\mathrm{cm}^{-1}\right): 3541,3063,3028,2854,1595,1452,1130,1026,987,920,872,844 ;{ }^{1} \mathrm{H}$ NMR $(400$ $\left.\mathrm{MHz}, \mathrm{CDCl}_{3}\right) \delta(\mathrm{ppm}):-0.03(\mathrm{~s}, 9 \mathrm{H}), 3.21(\mathrm{dd}, J=13.8,10.7 \mathrm{~Hz}, 1 \mathrm{H}), 3,29$ (dd, $\left.J=13.8,3.9 \mathrm{~Hz}, 1 \mathrm{H}\right)$, $3.78(\mathrm{~s}, 3 \mathrm{H}), 4.39$ (dd, $J=10.6,4.0 \mathrm{~Hz}, 1 \mathrm{H}), 5.47$ (s, 1H), $6.54(\mathrm{dd}, J=8.0,2.0 \mathrm{~Hz}, 1 \mathrm{H}), 6.58(\mathrm{~d}, J=$ $8.4 \mathrm{~Hz}, 1 \mathrm{H}), 6.90-7.06(\mathrm{~m}, 4 \mathrm{H}), 7.06-7.12(\mathrm{~m}, 3 \mathrm{H}), 7.16-7.22(\mathrm{~m}, 3 \mathrm{H}), 7.24-7.30(\mathrm{~m}, 1 \mathrm{H}) ;{ }^{13} \mathrm{C} \mathrm{NMR}$ $\left(100 \mathrm{MHz}, \mathrm{CDCl}_{3}\right) \delta(\mathrm{ppm}):-0.5,37.9,55.6,75.2,109.4,116.1,120.6,123.3,125.6,127.9,128.0$, 128.3, 129.1, 131.5, 139.3, 144.6, 145.6, 152.6; MS (EI, 70 eV): m/z (\%) = 227 (100), 407 (0.6); Anal. Calcd for $\mathrm{C}_{24} \mathrm{H}_{29} \mathrm{NO}_{3} \mathrm{Si}$ : C, 70.72; H,7.17; N, 3.44. Found: C, 70.48; H, 7.04; N, 3.25.

\section{4h: N-phenyl-N-(2-phenyl-1-(p-tolyl)ethyl)-O-(trimethylsilyl)hydroxylamine}




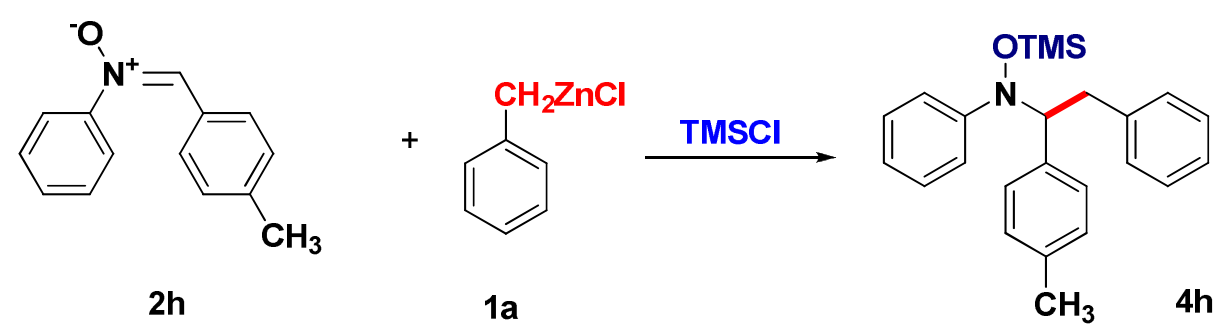

According to general procedure A: A solution of TMSCl $(0.65 \mathrm{~g}, 0.78 \mathrm{ml}, 6.0 \mathrm{mmol})$ in THF $(5 \mathrm{~mL})$ was added slowly to a mixture of N-phenyl-C-(methylphenyl) nitrone $\mathbf{2 h}(0.63 \mathrm{~g}, 3.0 \mathrm{mmol})$, benzylzinc chloride $(4.5 \mathrm{mmol})$ in $10 \mathrm{ml}$ of THF under room temperature. The reaction mixture was stirred at this temperature for half an hour. The pure product was obtained as colorless oil $(0.86 \mathrm{~g}, 2.28$ mmol, 76\%) after purification on silica gel (50:1 petroleum ether : EtOAc).

IR $(\mathrm{KBr}) \vee\left(\mathrm{cm}^{-1}\right): 3027,2958,2924,1595,1487,1250,879,843 ;{ }^{1} \mathrm{H}$ NMR (400 MHz, $\left.\mathrm{CDCl}_{3}\right) \delta$ (ppm): 0.31 (s, 9H), 2.27 (s, 3H), 3.25-3.45 (m, 2H), 4.49-4.58 (m, 1H), 6.90-7.40 (m, 14H); ${ }^{13} \mathrm{C}$ NMR $\left(100 \mathrm{MHz}, \mathrm{CDCl}_{3}\right) \delta(\mathrm{ppm}):-0.6,22.5,36.1,75.7,120.5,123.4,125.7,127.9,129.0,129.9,133.6$, 135.1, 137.2, 139.1, 141.6, 152.4. MS (EI, $70 \mathrm{eV}): \mathrm{m} / \mathrm{z}(\%)=195(100), 375\left(0.58,[\mathrm{M}]^{+}\right)$. Anal. Calcd for $\mathrm{C}_{24} \mathrm{H}_{29} \mathrm{NOSi}$ : C, 76.75; H,7.78; N, 3.73. Found: C, 76.47; H, 7.84; N, 3.42.

\section{4i: N-phenyl-N-(2-phenyl-1-(thiophen-3-yl)ethyl)-O-(trimethylsilyl)hydroxylamine}

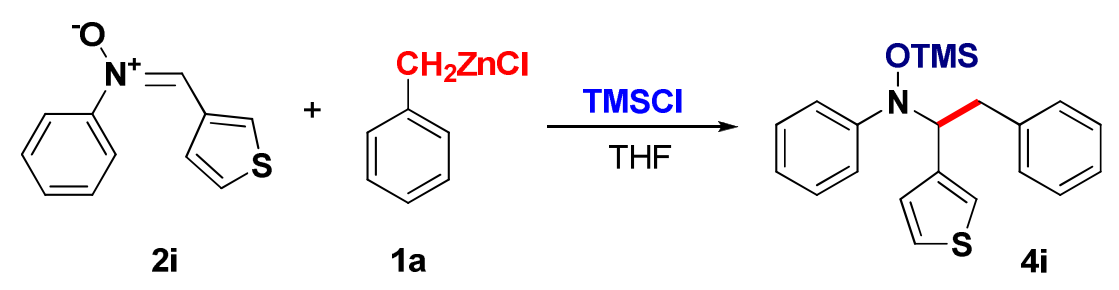

According to general procedure A: A solution of TMSCl $(0.65 \mathrm{~g}, 0.78 \mathrm{ml}, 6.0 \mathrm{mmol})$ in THF $(5 \mathrm{~mL})$ was added slowly to a mixture of N-phenyl-C-(3-thienyl) nitrone $2 \mathbf{i}(0.61 \mathrm{~g}, 3.0 \mathrm{mmol})$, benzylzinc chloride $(4.5 \mathrm{mmol})$ in $10 \mathrm{ml}$ of THF under room temperature. The reaction mixture was stirred at this temperature for half an hour. The pure product was obtained as colorless oil $(0.88 \mathrm{~g}, 2.4 \mathrm{mmol}, 80 \%)$ after purification on silica gel (50:1 petroleum ether : EtOAc).

IR $(\mathrm{KBr}) \vee\left(\mathrm{cm}^{-1}\right): 3028,2959,1596,1488,1452,1304,1250,1203,1080,925,878 ;{ }^{1} \mathrm{H}$ NMR $(400$ $\left.\mathrm{MHz}, \mathrm{CDCl}_{3}\right) \delta(\mathrm{ppm}): 0.20$ (s, 9H), 3.34 (dd, $\left.J=13.6,10.8 \mathrm{~Hz}, 1 \mathrm{H}\right), 3.56(\mathrm{dd}, J=13.6,3.6, \mathrm{~Hz}, 1 \mathrm{H})$, $4.90(\mathrm{dd}, J=10.8,4.0, \mathrm{~Hz}, 1 \mathrm{H}), 6.71(\mathrm{~s}, 1 \mathrm{H}), 6.86$ (dd, $J=5.2,3.6 \mathrm{~Hz}, 1 \mathrm{H}), 7.10$ (t, $J=13.2, \mathrm{~Hz}, 1 \mathrm{H})$, $7.19(\mathrm{t}, J=7.6 \mathrm{~Hz}, 2 \mathrm{H}), 7.20-7.26(\mathrm{~m}, 6 \mathrm{H}), 7.26-7.34(\mathrm{~m}, 2 \mathrm{H}) ;{ }^{13} \mathrm{C} \mathrm{NMR}\left(100 \mathrm{MHz}, \mathrm{CDCl}_{3}\right) \delta(\mathrm{ppm})$ : 0.8, 33.4, 78.7, 116.7, 119.9, 123.5, 125.9, 126.2, 128.2, 128.4, 129.1, 131.8, 133.6, 134.6, 136.7, 138.1, 152.4. MS (EI, $70 \mathrm{eV}): \mathrm{m} / \mathrm{z}(\%)=187(100), 367\left(0.2,[\mathrm{M}]^{+}\right)$; Anal. Calcd for $\mathrm{C}_{21} \mathrm{H}_{25} \mathrm{NOSSi}$ C, 68.62; H, 6.86; N, 3.81; S, 8.72. Found: C, 68.36; H, 6.64; N, 3.48; S, 7.47. 4j: N-(1-(furan-2-yl)-2-phenylethyl)-N-phenyl-O-(trimethylsilyl)hydroxylamine 


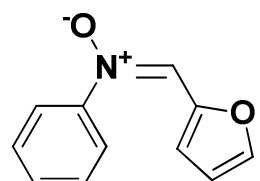

2j

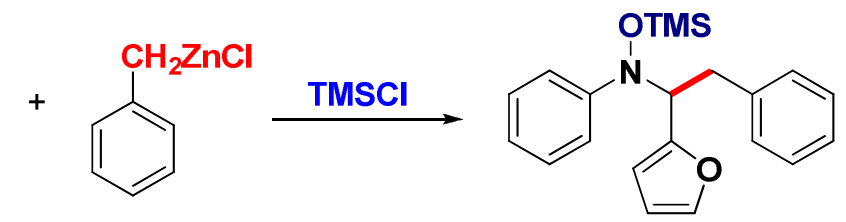

1a

4j

According to general procedure A: A solution of TMSCl $(0.65 \mathrm{~g}, 0.78 \mathrm{ml}, 6.0 \mathrm{mmol})$ in THF $(5 \mathrm{~mL})$ was added slowly to a mixture of N-phenyl-C-(2-furanyl) nitrone $2 \mathbf{j}$ (0.56 g, $3.0 \mathrm{mmol})$, benzylzinc chloride $(4.5 \mathrm{mmol})$ in $10 \mathrm{ml}$ of THF under room temperature. The reaction mixture was stirred at this temperature for half an hour. The pure product was obtained as yellowish oil (0.78 g, $2.4 \mathrm{mmol}, 74 \%)$ after purification on silica gel (50:1 petroleum ether : EtOAc).

IR $(\mathrm{KBr}) \vee\left(\mathrm{cm}^{-1}\right): 2958,2926,1590,1486,1452,1250,878,697 ;{ }^{1} \mathrm{H}$ NMR (400 MHz, $\left.\mathrm{CDCl}_{3}\right) \delta$ (ppm): -0.19 (s, 9H), 3.00-3.07 (m, 2H), 4.40 (q, $J=7.2 \mathrm{~Hz}, 1 \mathrm{H}), 5.95$ (d, $J=3.41 \mathrm{~Hz}, 1 \mathrm{H}), 6.03-6.05$ $(\mathrm{m}, 1 \mathrm{H}), 6.75-7.00(\mathrm{~m}, 8 \mathrm{H}), 7.03-7.15(\mathrm{~m}, 2 \mathrm{H}), 7.39-7.41(\mathrm{~m}, 2 \mathrm{H}) ;{ }^{13} \mathrm{C} \mathrm{NMR}\left(100 \mathrm{MHz}, \mathrm{CDCl}_{3}\right) \delta$ (ppm): -0.8, 33.4, 69.5, 119.9, 123.5, 125.9, 126.2, 128.2, 128.8, 129.1, 131.8, 133.6, 134.6, 136.7, 138.1, 152.4. MS (EI, $70 \mathrm{eV}): \mathrm{m} / \mathrm{z}(\%)=171(100), 351\left(0.6,[\mathrm{M}]^{+}\right)$; Anal. Calcd for $\mathrm{C}_{21} \mathrm{H}_{25} \mathrm{NO}_{2} \mathrm{Si}: \mathrm{C}$, 71.75; H, 7.17; N, 3.98. Found: C, 71.57; H, 6.94; N, 3.88.

\section{4k: 4-(2-(4-chlorophenyl)-1-(phenyl((trimethylsilyl)oxy)amino)ethyl)phenol}

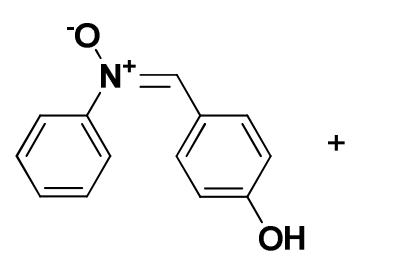

2k

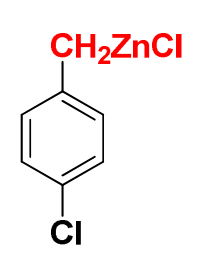

1b
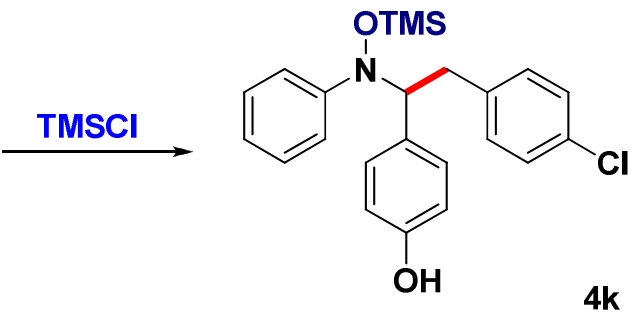

According to general procedure A: A solution of TMSCl $(0.65 \mathrm{~g}, 0.78 \mathrm{ml}, 6.0 \mathrm{mmol})$ in THF $(5 \mathrm{~mL})$ was added slowly to a mixture of N-phenyl-C-(4-hydroxyphenyl) nitrone $\mathbf{2 k}(0.64 \mathrm{~g}, 3.0 \mathrm{mmol})$, 4chlorobenzylzinc chloride $(4.5 \mathrm{mmol})$ in $10 \mathrm{ml}$ of THF under room temperature. The reaction mixture was stirred at this temperature for half an hour. The pure product was obtained as yellowish oil $(0.99 \mathrm{~g}$, $2.4 \mathrm{mmol}, 56 \%)$ after purification on silica gel (20:1 petroleum ether : EtOAc).

IR $(\mathrm{KBr}) \vee\left(\mathrm{cm}^{-1}\right): 3334,3029,2959,2926,1597,1489,1452,921,842 ;{ }^{1} \mathrm{H} \mathrm{NMR}\left(400 \mathrm{MHz}, \mathrm{CDCl}_{3}\right) \delta$ (ppm): -0.05 (s, 9H), $3.21(\mathrm{dd}, J=13.8,10.7 \mathrm{~Hz}, 1 \mathrm{H}), 3,29(\mathrm{dd}, J=13.8,3.9 \mathrm{~Hz}, 1 \mathrm{H}), 4.40(\mathrm{dd}, J=$ 10.6, 4.0 Hz, 1H), 5.40 (brs, 1H), 6.63 (dd, $J=4.8,2.0 \mathrm{~Hz}, 1 \mathrm{H}), 6.92-7.12$ (m, $11 \mathrm{H}), 7.19$ (dd, $J=8.4$, $7.2 \mathrm{~Hz}, 1 \mathrm{H}) ;{ }^{13} \mathrm{C}$ NMR $\left(100 \mathrm{MHz}, \mathrm{CDCl}_{3}\right) \delta(\mathrm{ppm}):-0.5,36.1,67.9,75.2,114.4,120.7,123.4,125.6$, $127.9,128.0,129.2,130.3,131.3,139.4,152.6,154.9$. MS (EI, $70 \mathrm{eV}): \mathrm{m} / \mathrm{z}(\%)=197.15(100), 412$ 
(0.59, $\left.[\mathrm{M}+\mathrm{H}]^{+}\right)$; Anal. Calcd for $\mathrm{C}_{23} \mathrm{H}_{26} \mathrm{ClNO}_{2} \mathrm{Si}$ : C, 67.05; H,6.36; N, 3.40. Found: C, 66.83; H, 6.12; $\mathrm{N}, 3.16$.

\section{4l: N-(2-(4-chlorophenyl)-1-(thiophen-3-yl)ethyl)-N-phenyl-O-(trimethylsilyl)hydroxylamine}<smiles>O=[N+]([O-])/C=C/c1ccsc1</smiles>

According to general procedure A: A solution of TMSCl $(0.65 \mathrm{~g}, 0.78 \mathrm{ml}, 6.0 \mathrm{mmol})$ in THF (5 mL) was added slowly to a mixture of N-phenyl-C-(3-thienyl) nitrone $2 \mathbf{i}(0.61 \mathrm{~g}, 3.0 \mathrm{mmol})$, 4chlorobenzylzinc chloride $(4.5 \mathrm{mmol})$ in $10 \mathrm{ml}$ of THF under room temperature. The reaction mixture was stirred at this temperature for half an hour. The pure product was obtained as yellowish syrup (0.95 g, $2.4 \mathrm{mmol}, 79 \%)$ after purification on silica gel (50:1 petroleum ether : EtOAc).

IR $(\mathrm{KBr}) \vee\left(\mathrm{cm}^{-1}\right): 3067,3029,2959,1593,1490,1449,1407,1250,1204,1094,880,847,766,698$; ${ }^{1} \mathrm{H}$ NMR (400 MHz, $\left.\mathrm{CDCl}_{3}\right) \delta(\mathrm{ppm}): 0.16$ (s, 9H), 3.26 (dd, $\left.J=13.6,10.8 \mathrm{~Hz}, 1 \mathrm{H}\right), 3.41$ (dd, $J=13.2$, $3.6 \mathrm{~Hz}, 1 \mathrm{H}), 4.49(\mathrm{dd}, J=14.4,3.6 \mathrm{~Hz}, 1 \mathrm{H}), 6.50(\mathrm{~s}, 1 \mathrm{H}), 6.95-7.25(\mathrm{~m}, 12 \mathrm{H}) ;{ }^{13} \mathrm{C} \mathrm{NMR}(100 \mathrm{MHz}$, $\left.\mathrm{CDCl}_{3}\right) \delta(\mathrm{ppm}):-0.5,38.6,71.6,120.2,123.6,125.2,125.7,127.2,128.2,130.4,131.7,137.4,140.3$, 152.1. MS (EI, $70 \mathrm{eV}): \mathrm{m} / \mathrm{z}(\%)=276(100), 401\left(0.97,[\mathrm{M}]^{+}\right)$; Anal. Calcd for $\mathrm{C}_{21} \mathrm{H}_{24} \mathrm{ClNOSSi}$ : C, 62.74; H,6.02; N, 3.48. Found: C, 62.88; H, 5.78; N, 3.16.

\section{4m: N-(2-(4-chlorophenyl)-1-phenylethyl)-N-phenyl-O-(trimethylsilyl)hydroxylamine}

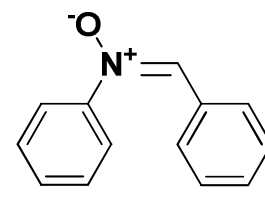

2a

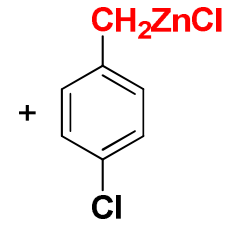

$1 \mathrm{~b}$

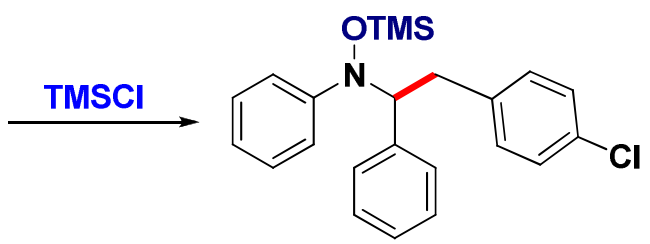

$4 \mathrm{~m}$

According to general procedure A: A solution of TMSCl $(0.65 \mathrm{~g}, 0.78 \mathrm{ml}, 6.0 \mathrm{mmol})$ in THF (5 mL) was added slowly to a mixture of N-phenyl-C-phenyl nitrone 2a (0.59 g, $3.0 \mathrm{mmol})$, 4chlorobenzylzinc chloride $(4.5 \mathrm{mmol})$ in $10 \mathrm{ml}$ of THF under room temperature. The reaction mixture was stirred at this temperature for half an hour. The pure product was obtained as yellowish syrup $(0.90$ g, $2.28 \mathrm{mmol}, 76 \%)$ after purification on silica gel (50:1 petroleum ether : EtOAc).

IR $(\mathrm{KBr}) \vee\left(\mathrm{cm}^{-1}\right):$ 3063, 3028, 2967, 2926, 1596, 1489, 1452, 1406, 1375, 1300, 1256, 1207, 1169 , 1095, 1028, 966, 930, 874; ${ }^{1} \mathrm{H}$ NMR (400 MHz, $\left.\mathrm{CDCl}_{3}\right) \delta(\mathrm{ppm}):-0.086(\mathrm{~s}, 9 \mathrm{H}), 3.18-3.30(\mathrm{~m}, 2 \mathrm{H})$, $4.59(\mathrm{q}, J=4.8 \mathrm{~Hz}, 1 \mathrm{H}), 6.86(\mathrm{~d}, J=8.0 \mathrm{~Hz}, 2 \mathrm{H}), 7.04(\mathrm{~d}, \mathrm{~J}=8.4 \mathrm{~Hz}, 2 \mathrm{H}), 7.10(\mathrm{~d}, J=8.0 \mathrm{~Hz}, 2 \mathrm{H})$, 
7.15-7.25 (m, 8H); ${ }^{13} \mathrm{C}$ NMR $\left(100 \mathrm{MHz}, \mathrm{CDCl}_{3}\right) \delta(\mathrm{ppm}):-0.6,35.3,75.8,120.7,123.6,127.5,127.6$, 128.1, 128.1, 130.0, 130.5, 131.4, 137.7, 138.0, 152.5. MS (EI, 70 eV): m/z (\%): 270 (100), 395 (0.23, $\left.[\mathrm{M}]^{+}\right)$; Anal. Calcd for $\mathrm{C}_{23} \mathrm{H}_{26} \mathrm{ClNOSi}$ : C, 69.76; H,6.62; N, 3.54. Found: C, 69.58; H, 6.42; N, 3.43.

4n: N-(2-(4-chlorophenyl)-1-(4-methoxyphenyl)ethyl)-N-phenyl-O- (trimethylsilyl)hydroxylamine<smiles>COc1ccc(/C=[N+](/[O-])c2ccccc2)cc1</smiles>

2b<smiles>ClCc1ccc(Cl)cc1</smiles>

$1 \mathrm{~b}$

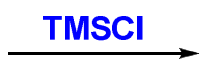<smiles>COc1ccc(C(Cc2ccc(Cl)cc2)N(OC)c2ccccc2)cc1</smiles>

According to general procedure A: A solution of TMSCl $(0.65 \mathrm{~g}, 0.78 \mathrm{ml}, 6.0 \mathrm{mmol})$ in THF $(5 \mathrm{~mL})$ was added slowly to a mixture of N-phenyl-C-(4-methoxyphenyl) nitrone $\mathbf{2 b}(0.68 \mathrm{~g}, 3.0 \mathrm{mmol})$, 4chlorobenzylzinc chloride $(4.5 \mathrm{mmol})$ in $10 \mathrm{ml}$ of THF under room temperature. The reaction mixture was stirred at this temperature for half an hour. The pure product was obtained as colorless syrup (0.99 g, $2.34 \mathrm{mmol}, 78 \%)$ after purification on silica gel (30:1 petroleum ether : EtOAc).

IR $(\mathrm{KBr}) \vee\left(\mathrm{cm}^{-1}\right): 2957,2926,1596,1489,1452,1406,1251,1207,1169,1095,1028,966,874,844$; ${ }^{1} \mathrm{H}$ NMR (400 MHz, $\mathrm{CDCl}_{3}$ ) $\delta$ (ppm): 0.10 (s, 9H), 3.37 (dd, $\left.J=14.0,3.2 \mathrm{~Hz}, 1 \mathrm{H}\right), 3.48$ (dd, $J=13.6$, $3.6 \mathrm{~Hz}, 1 \mathrm{H}), 3.88$ (s, 3H), $4.60(\mathrm{dd}, J=10.8,4.0 \mathrm{~Hz}, 1 \mathrm{H}), 6.86(\mathrm{dd}, J=6.4,2.0 \mathrm{~Hz}, 2 \mathrm{H}), 7.10-7.22(\mathrm{~m}$, 4H), 7.22-7.30 (m, 5H), $7.36(\mathrm{dd}, J=8.4,7.2 \mathrm{~Hz}, 2 \mathrm{H}) ;{ }^{13} \mathrm{C} \mathrm{NMR}\left(100 \mathrm{MHz}, \mathrm{CDCl}_{3}\right) \delta$ (ppm): -0.5, 36.1, 55.0, 75.2, 112.8, 120.6, 123.3, 125.6, 127.9, 128.0, 129.1, 130. 3, 131.0, 139.4, 152.6, 158.7. MS (ESI, m/z ): $425.8[\mathrm{M}]^{+}$; Anal. Calcd for $\mathrm{C}_{24} \mathrm{H}_{28} \mathrm{ClNO}_{2} \mathrm{Si}$ : C, 67.66; H,6.62; N, 3.29. Found: C, 67.45; H, $6.38 ; \mathrm{N}, 3.08$.

\section{0:N-(2-(4-chlorophenyl)-1-(3,4,5-trimethoxyphenyl)ethyl)-N-phenyl-O-} (trimethylsilyl)hydroxylamine
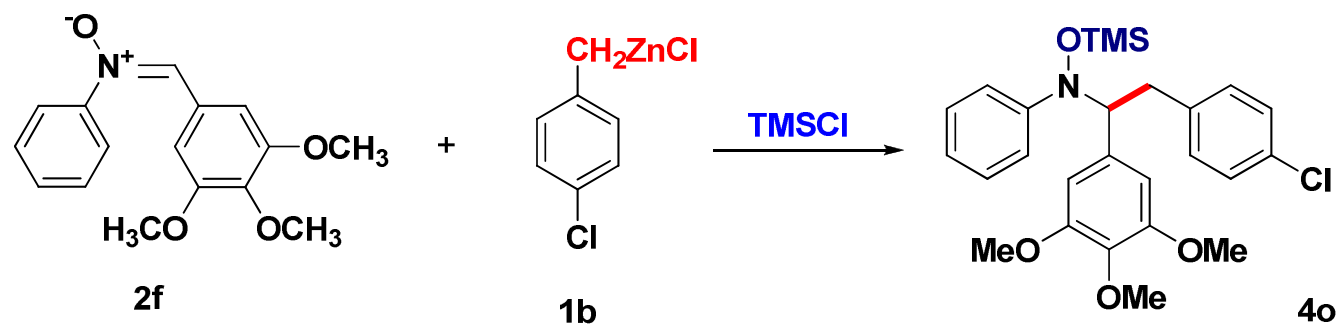
According to general procedure A: A solution of TMSCl $(0.65 \mathrm{~g}, 0.78 \mathrm{ml}, 6.0 \mathrm{mmol})$ in THF $(5 \mathrm{~mL})$ was added slowly to a mixture of N-phenyl-C-(3, 4, 5-trimethoxyphenyl) nitrone $\mathbf{2 f}(0.68 \mathrm{~g}, 3.0 \mathrm{mmol})$, 4-chlorobenzylzinc chloride $(4.5 \mathrm{mmol})$ in $10 \mathrm{ml}$ of $\mathrm{THF}$ under room temperature. The reaction mixture was stirred at this temperature for half an hour. The pure product was obtained as colorless syrup (1.06 g, $2.34 \mathrm{mmol}, 78 \%)$ after purification on silica gel (20:1 petroleum ether : EtOAc).

IR $(\mathrm{KBr}) \vee\left(\mathrm{cm}^{-1}\right): 3575,3516,3004,2961,1593,1491,1423,1362,1223,1128,1093,1013,924,883$, 846; ${ }^{1} \mathrm{H}$ NMR $\left(400 \mathrm{MHz}, \mathrm{CDCl}_{3}\right) \delta(\mathrm{ppm}):-0.10(\mathrm{~s}, 9 \mathrm{H}), 3.12(\mathrm{dd}, J=13.6,10.8 \mathrm{~Hz}, 1 \mathrm{H}), 3.20(\mathrm{dd}, J=$ 14.0, $4.0 \mathrm{~Hz}, 1 \mathrm{H}), 3.59$ (s, 6H), 3.68 (s, 3H), 4.33 (dd, J = 6.4, $4.0 \mathrm{~Hz}, 1 \mathrm{H}), 6.31$ (s, $2 \mathrm{H}), 6.82-6.96$ (m, 2H), 6.98-7.04 (m, 4H), $7.09(\mathrm{t}, J=8.4 \mathrm{~Hz}, 2 \mathrm{H}) ;{ }^{13} \mathrm{C} \mathrm{NMR}\left(100 \mathrm{MHz}, \mathrm{CDCl}_{3}\right) \delta(\mathrm{ppm}):-0.6,35.5,55.9$, 60.6, 75.7, 107.2, 120.6, 123.6, 128.0, 130.3, 133.3, 137.6, 152.2. MS (EI, $70 \mathrm{eV}): \mathrm{m} / \mathrm{z}(\%)=305(100)$, $485\left(0.2,[\mathrm{M}]^{+}\right)$; Anal. Calcd for $\mathrm{C}_{26} \mathrm{H}_{32} \mathrm{ClNO}_{4} \mathrm{Si}$ : C, 64.24; H, 6.64; N, 2.88. Found: C, 63.96; H, 6.34; $\mathrm{N}, 2.64$.

\section{4p:}

N-(2-(4-chlorophenyl)-1-(2,4-dichlorophenyl)ethyl)-N-phenyl-O-(trimethylsilyl) hydroxylamine

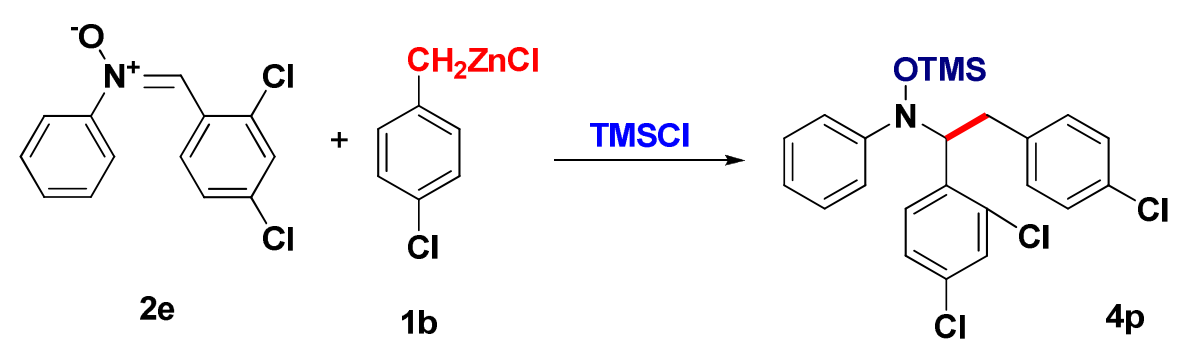

According to general procedure A: A solution of TMSCl $(0.65 \mathrm{~g}, 0.78 \mathrm{ml}, 6.0 \mathrm{mmol})$ in THF $(5 \mathrm{~mL})$ was added slowly to a mixture of N-phenyl-C-(2,4-dichlorophenyl) nitrone $2 \mathbf{e}(0.68 \mathrm{~g}, 3.0 \mathrm{mmol})$, 4chlorobenzylzinc chloride $(4.5 \mathrm{mmol})$ in $10 \mathrm{ml}$ of THF under room temperature. The reaction mixture was stirred at this temperature for half an hour. The pure product was obtained as colorless syrup (1.14 $\mathrm{g}, 2.46 \mathrm{mmol}, 82 \%)$ after purification on silica gel (30:1 petroleum ether : EtOAc).

IR $(\mathrm{KBr}) \vee\left(\mathrm{cm}^{-1}\right): 2958,2926,1591,1489,1452,1250,897,848 ;{ }^{1} \mathrm{H}$ NMR (400 MHz, $\left.\mathrm{CDCl}_{3}\right) \delta$ (ppm): 0.00 (s, 9H), 3.33 (dd, $J=14.0,4.0 \mathrm{~Hz}, 1 \mathrm{H}), 3.48$ (dd, $J=14.0,11.2 \mathrm{~Hz}, 1 \mathrm{H}), 5.31$ (dd, $J=11.6$, $4.4 \mathrm{~Hz}, 1 \mathrm{H}), 7.02(\mathrm{~d}, J=8.4 \mathrm{~Hz}, 2 \mathrm{H}), 7.35-7.54(\mathrm{~m}, 3 \mathrm{H}), 7.56-7.61(\mathrm{~m}, 6 \mathrm{H}), 7.76(\mathrm{~d}, J=8.4 \mathrm{~Hz}, 1 \mathrm{H})$;

${ }^{13} \mathrm{C} \mathrm{NMR}\left(100 \mathrm{MHz}, \mathrm{CDCl}_{3}\right) \delta$ (ppm): -0.9, 33.0, 69.6, 119.9, 123.7, 126.2, 128.3, 128.4, 129.8, 131.7, 133.8, 134.3, 136.6, 152.3, 130.2. MS (EI, $70 \mathrm{eV}): \mathrm{m} / \mathrm{z}(\%)=338(100), 463\left(3.9,[\mathrm{M}]^{+}\right)$; Anal. Calcd for $\mathrm{C}_{23} \mathrm{H}_{24} \mathrm{Cl}_{3} \mathrm{NOSi}$ : C, 59.42; H, 5.20; N, 3.01. Found: C, 59.24; H, 4.96; N, 2.68.

\section{4q: (S)-4-(2-(4-chlorophenyl)-1-(phenyl((trimethylsilyl)oxy)amino)ethyl)phenol}




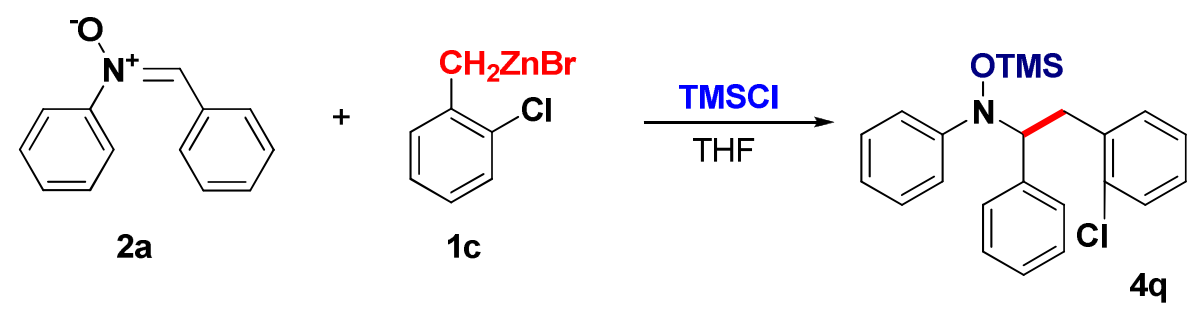

According to general procedure A: A solution of TMSCl $(0.65 \mathrm{~g}, 0.78 \mathrm{ml}, 6.0 \mathrm{mmol})$ in THF $(5 \mathrm{~mL})$ was added slowly to a mixture of N-phenyl-C-phenyl nitrone 2a $(0.59 \mathrm{~g}, 3.0 \mathrm{mmol})$, 2chlorobenzylzinc bromide $(4.5 \mathrm{mmol})$ in $10 \mathrm{ml}$ of THF under room temperature. The reaction mixture was stirred at this temperature for half an hour. The pure product was obtained as colorless syrup (0.78 g, $1.98 \mathrm{mmol}, 66 \%)$ after purification on silica gel (50:1 petroleum ether : EtOAc).

IR $(\mathrm{KBr}) \vee\left(\mathrm{cm}^{-1}\right): 3334,3030,2959,1597,1490,1453,1251,921,842 ;{ }^{1} \mathrm{H} \mathrm{NMR}\left(400 \mathrm{MHz}, \mathrm{CDCl}_{3}\right) \delta$ (ppm): -0.08 (s, 9H), $3.23(\mathrm{dd}, J=13.79,3.5 \mathrm{~Hz}, 1 \mathrm{H}), 3.65(\mathrm{dd}, J=13.80,11.36 \mathrm{~Hz}, 1 \mathrm{H}), 5.27(\mathrm{dd}, J=$ $11.2,3.6 \mathrm{~Hz}, 1 \mathrm{H}), 6.72(\mathrm{~d}, J=8.0 \mathrm{~Hz}, 1 \mathrm{H}), 6.95-7,15(\mathrm{~m}, 7 \mathrm{H}), 7.20-7.40(\mathrm{~m}, 5 \mathrm{H}), 7.68$ (dd, $J=7.7,1.6$ $\mathrm{Hz}, 1 \mathrm{H}) ;{ }^{13} \mathrm{C} \mathrm{NMR}\left(100 \mathrm{MHz}, \mathrm{CDCl}_{3}\right) \delta(\mathrm{ppm}):-0.4,33.6,66.9,117.7,119.6,120.1,122.6,125.4$, 127.9, 128.0, 129.2, 130.7, 139.6, 153.3, 154.4. MS (EI, $70 \mathrm{eV}): \mathrm{m} / \mathrm{z}(\%)=197$ (100), $395(0.02$, $[\mathrm{M}]+)$; Anal. Calcd for $\mathrm{C}_{23} \mathrm{H}_{26}$ ClNOSi: C, 69.76; H, 6.62; N, 3.54. Found: C, 69.54; H, 6.46; N, 3.44.

4r: N-(1-(2-chlorophenyl)-2-(4-chlorophenyl)ethyl)-N-phenyl-O-(trimethylsilyl)hydroxylamine

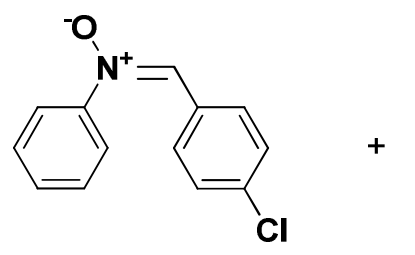

2d

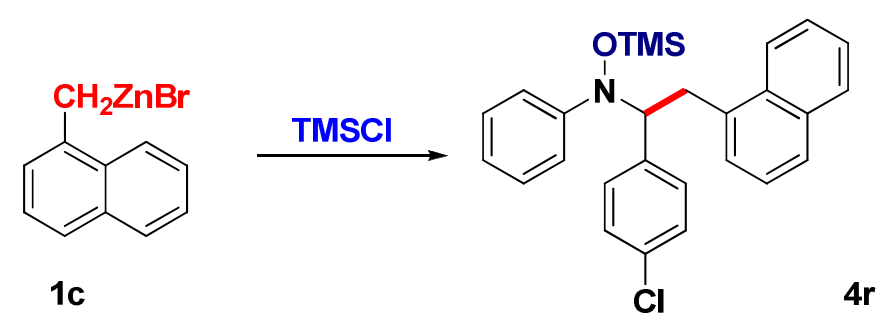

According to general procedure A: A solution of TMSCl $(0.65 \mathrm{~g}, 0.78 \mathrm{ml}, 6.0 \mathrm{mmol})$ in THF $(5 \mathrm{~mL})$ was added slowly to a mixture of N-phenyl-C-(4-chlorophenyl) nitrone 2d (0.70 g, $3.0 \mathrm{mmol}), 1$ naphthylzinc bromide $(4.5 \mathrm{mmol})$ in $10 \mathrm{ml}$ of THF under room temperature. The reaction mixture was stirred at this temperature for half an hour. The pure product was obtained as yellowish syrup (0.83 $\mathrm{g}$, $1.86 \mathrm{mmol}, 62 \%)$ after purification on silica gel (30:1 petroleum ether : EtOAc).

IR $(\mathrm{KBr}) \vee\left(\mathrm{cm}^{-1}\right): 2923,2852,1591,1489,1456,1420,1330,1244,1184,1128,1011,928,887 ;{ }^{1} \mathrm{H}$ NMR (400 MHz, $\left.\mathrm{CDCl}_{3}\right) \delta(\mathrm{ppm}):-0.06(\mathrm{~s}, 9 \mathrm{H}), 3.26$ (dd, $\left.J=14.4,3.2 \mathrm{~Hz}, 1 \mathrm{H}\right), 3.50$ (dd, $J=14.4,12$ $\mathrm{Hz}, 1 \mathrm{H}), 5.44(\mathrm{dt}, J=10.8,3.2 \mathrm{~Hz}, 1 \mathrm{H}), 6.94(\mathrm{dd}, J=6.8,1.2 \mathrm{~Hz}, 2 \mathrm{H}), 7.05-7.15(\mathrm{~m}, 4 \mathrm{H}), 7.21(\mathrm{t}, J=$ 7.6Hz, 1H), 7.25-7.50 (m, 8H), $7.72(\mathrm{~d}, J=7.6 \mathrm{~Hz}, 1 \mathrm{H}) ;{ }^{13} \mathrm{C} \mathrm{NMR}\left(100 \mathrm{MHz}, \mathrm{CDCl}_{3}\right) \delta(\mathrm{ppm}):-0.8$, $36.9,69.9,119.7,123.3,125.9,128.3,129.4,129.7,130.2$, 131.4, 135.6, 137.0, 152.5. MS (EI, 70 eV): 
m/z $(\%)=304(100), 445\left(0.04,[\mathrm{M}]^{+}\right)$; Anal. Calcd for $\mathrm{C}_{27} \mathrm{H}_{28} \mathrm{ClNOSi}$ : C,72.70; H,6.33; N, 3.14. Found: C, 72.45; H, 6.08; N, 2.86.

6a: (S)-N-(1-(4-methoxyphenyl)-2-phenylethyl)-N-phenyl-O-(trimethylsilyl)hydroxylamine<smiles>[O-][N+](=Cc1ccc(F)cc1)c1ccccc1</smiles><smiles>CON(c1ccc(C(C)(C)C)cc1)C(c1ccccc1)c1ccc(F)cc1</smiles>

According to general procedure A: A solution of TMSCl $(0.65 \mathrm{~g}, 0.78 \mathrm{ml}, 6.0 \mathrm{mmol})$ in THF $(5 \mathrm{~mL})$ was added slowly to a mixture of N-phenyl-C-(4-fluorophenyl) nitrone 21 (0.65 g, $3.0 \mathrm{mmol})$, phenylzinc bromide $(4.5 \mathrm{mmol})$ in $10 \mathrm{ml}$ of THF under room temperature. The reaction mixture was stirred at this temperature for half an hour. The pure product was obtained as yellowish syrup $(0.81 \mathrm{~g}$, $2.22 \mathrm{mmol}, 74 \%)$ after purification on silica gel (30:1 petroleum ether : EtOAc).

IR $(\mathrm{KBr}) \vee\left(\mathrm{cm}^{-1}\right): 3459,2923,2852,1598,1506,1250,1222,1027,843,756 ;{ }^{1} \mathrm{H}$ NMR (400 MHz, $\left.\mathrm{CDCl}_{3}\right) \delta(\mathrm{ppm}):-0.14(\mathrm{~s}, 9 \mathrm{H}), 5.71(\mathrm{~s}, 1 \mathrm{H}), 6.82(\mathrm{dd}, J=3.6,2.0 \mathrm{~Hz}, 1 \mathrm{H}), 6.86(\mathrm{tt}, J=3.6,2.0 \mathrm{~Hz}, 1 \mathrm{H})$, 6.92-7.02 (m, 4H), 7.08-7.18 (m, 5H), $7.23(\mathrm{dd}, J=5.2,1.2 \mathrm{~Hz}, 1 \mathrm{H}), 7.42(\mathrm{dd}, J=8.4,6.0 \mathrm{~Hz}, 2 \mathrm{H}) ;{ }^{13} \mathrm{C}$ NMR $\left(100 \mathrm{MHz}, \mathrm{CDCl}_{3}\right) \delta(\mathrm{ppm}):-0.7,73.5,114.5,114.7,120.4,123.7,125.8,127.6,128.1,131.4$, 135.4, 140.6, 152.2, $162.2(\mathrm{~d}, J=245 \mathrm{~Hz}) ; \mathrm{MS}(\mathrm{ESI}, \mathrm{m} / \mathrm{z}): 366.0[\mathrm{M}+\mathrm{H}]^{+}$; Anal. Calcd for $\mathrm{C}_{22} \mathrm{H}_{24}$ FNOSi: C,72.29; H, 6.62; N, 3.83. Found: C, 72.54; H, 6.44; N, 3.74.

\section{6b: N-((2-chlorophenyl)(phenyl)methyl)-N-phenyl-O-(trimethylsilyl)hydroxylamine}<smiles>[O-]/[N+](=C/c1ccccc1Cl)c1ccccc1</smiles>

2c

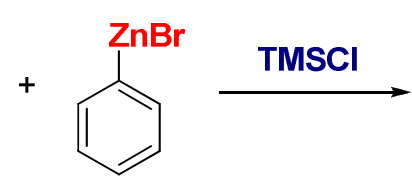

$7 a$<smiles>CON(c1ccccc1)C(c1ccccc1Cl)c1ccccc1Cl</smiles>

According to general procedure A: A solution of TMSCl $(0.65 \mathrm{~g}, 0.78 \mathrm{ml}, 6.0 \mathrm{mmol})$ in THF $(5 \mathrm{~mL})$ was added slowly to a mixture of N-phenyl-C-(2-chlorophenyl) nitrone $2 \mathrm{c}(0.70 \mathrm{~g}, 3.0 \mathrm{mmol})$, phenylzinc bromide $(4.5 \mathrm{mmol})$ in $10 \mathrm{ml}$ of THF under room temperature. The reaction mixture was stirred at this temperature for half an hour. The pure product was obtained as colorless syrup $(0.81 \mathrm{~g}$, $2.13 \mathrm{mmol}, 71 \%)$ after purification on silica gel (30:1 petroleum ether : EtOAc).

IR $(\mathrm{KBr}) \vee\left(\mathrm{cm}^{-1}\right): 3450,2957,2924,1593,1486,1444,1249,840,754,694 ;{ }^{1} \mathrm{H}$ NMR (400 MHz, $\left.\mathrm{CDCl}_{3}\right) \delta(\mathrm{ppm}):-0.22(\mathrm{~s}, 9 \mathrm{H}), 6.19(\mathrm{~s}, 1 \mathrm{H}), 6.88(\mathrm{tt}, J=7.6 \mathrm{~Hz}, 1 \mathrm{H}), 7.10-7.35(\mathrm{~m}, 10 \mathrm{H}), 7.47 \mathrm{dd}, J=$ 
8.0, $1.6 \mathrm{~Hz}, 2 \mathrm{H}), 7.77(\mathrm{dd}, J=8.0,2.0 \mathrm{~Hz}, 1 \mathrm{H}) ;{ }^{13} \mathrm{C} \mathrm{NMR}\left(100 \mathrm{MHz}, \mathrm{CDCl}_{3}\right) \delta(\mathrm{ppm}):-0.8,72.3$, $119.8,122.9$, 126.1, 127.2, 127.9, 128.1, 128.3, 129.3, 130.2, 132.1, 134.5, 137.8, 139.0, 152.6; MS (ESI, m/z ): $382.0[\mathrm{M}+\mathrm{H}]^{+}$; Anal. Calcd for $\mathrm{C}_{22} \mathrm{H}_{24} \mathrm{ClNOSi}$ : C,69.18; H, 6.33; N, 3.67. Found: C, 69.06; $\mathrm{H}, 6.23 ; \mathrm{N}, 3.42$.

6c: N-phenyl-N-(phenyl(3,4,5-trimethoxyphenyl)methyl)-O-(trimethylsilyl)hydroxylamine<smiles>COc1cc(/C=[N+](/[O-])c2ccccc2)cc(OC)c1OC</smiles><smiles>COc1cc(C(c2ccccc2)N(OC(C)C)c2ccccc2)cc(OC)c1OC</smiles>

According to general procedure A: A solution of TMSCl $(0.65 \mathrm{~g}, 0.78 \mathrm{ml}, 6.0 \mathrm{mmol})$ in THF $(5 \mathrm{~mL})$ was added slowly to a mixture of N-phenyl-C-(3,4,5-trimethoxyphenyl) nitrone $\mathbf{2 f}(0.86 \mathrm{~g}, 3.0 \mathrm{mmol})$, phenylzinc bromide $(4.5 \mathrm{mmol})$ in $10 \mathrm{ml}$ of THF under room temperature. The reaction mixture was stirred at this temperature for half an hour. The pure product was obtained as colorless syrup (1.19 g, $2.73 \mathrm{mmol}, 91 \%)$ after purification on silica gel (20:1 petroleum ether : EtOAc).

IR $(\mathrm{KBr}) \vee\left(\mathrm{cm}^{-1}\right): 2923,2852,1591,1456,1244,1128,842 ;{ }^{1} \mathrm{H}$ NMR $\left(400 \mathrm{MHz}, \mathrm{CDCl}_{3}\right) \delta(\mathrm{ppm})$ : 0.01(s, 9H), 3.87 (s, 6H), $3.92(\mathrm{~s}, 3 \mathrm{H}), 5.69$ (s, 1H), $6.75(\mathrm{~s}, 2 \mathrm{H}), 7.01-7.02(\mathrm{~m}, 1 \mathrm{H})$, 7.39-7.51 (m, 4H), 7.32-7.38 (m, 3H), $7.54(\mathrm{~d}, J=6.8 \mathrm{~Hz}, 2 \mathrm{H}) ;{ }^{13} \mathrm{C} \mathrm{NMR}\left(100 \mathrm{MHz}, \mathrm{CDCl}_{3}\right) \delta(\mathrm{ppm}):-0.5,55.9,76.7$, 120.6, 123.4, 127.1, 127.7, 127.9, 129.8, 135.1, 137.0, 139.7, 152.5, 152.7. MS (ESI, m/z): 436.3 [M$\mathrm{H}]^{-}$; Anal. Calcd for $\mathrm{C}_{25} \mathrm{H}_{31} \mathrm{NO}_{4} \mathrm{Si}: \mathrm{C}, 68.62 ; \mathrm{H}, 7.14 ; \mathrm{N}, 3.20$. Found: C, 68.34; H, 6.88; N, 3.16.

\section{6d: N-benzhydryl-N-benzyl-O-(trimethylsilyl)hydroxylamine}

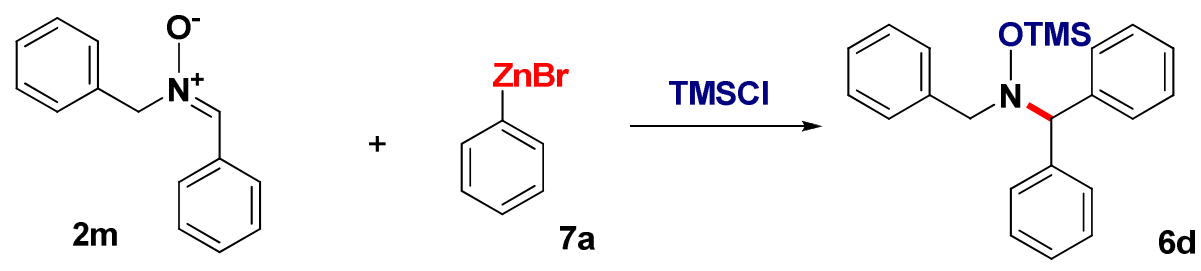

According to general procedure A: A solution of TMSCl $(0.65 \mathrm{~g}, 0.78 \mathrm{ml}, 6.0 \mathrm{mmol})$ in THF (5 mL) was added slowly to a mixture of N-benzyl-C-phenyl nitrone $2 \mathrm{~m}(0.63 \mathrm{~g}, 3.0 \mathrm{mmol})$, phenylzinc bromide $(4.5 \mathrm{mmol})$ in $10 \mathrm{ml}$ of THF under room temperature. The reaction mixture was stirred at this temperature for half an hour. The pure product was obtained as colorless syrup (0.79 g, $2.19 \mathrm{mmol}$, $73 \%)$ after purification on silica gel (50:1 petroleum ether : EtOAc).

IR $(\mathrm{KBr}) \vee\left(\mathrm{cm}^{-1}\right): 3028,2924,2851,1598,1456,1247,1126,844 ;{ }^{1} \mathrm{H}$ NMR $\left(400 \mathrm{MHz}, \mathrm{CDCl}_{3}\right) \delta$ (ppm): -0.08 (s, 9H), 4.04 (d, $J=14.8 \mathrm{~Hz}, 1 \mathrm{H}), 4.24$ (d, $J=14.8 \mathrm{~Hz}, 1 \mathrm{H}), 6.38(\mathrm{~s}, 1 \mathrm{H}), 6.95-7.26$ (m, $15 \mathrm{H}) ;{ }^{13} \mathrm{C}$ NMR $\left(100 \mathrm{MHz}, \mathrm{CDCl}_{3}\right) \delta(\mathrm{ppm}):-1.1,65.2,69.6,119.6,123.2,125.7,126.2,128.0,128.1$, 
128.0, 129.5, 132.2, 134.7, 137.6, 138.6, 152.4. MS (ESI, m/z): $361.8[\mathrm{M}+\mathrm{H}]^{+}$, Anal. Calcd for $\mathrm{C}_{23} \mathrm{H}_{27} \mathrm{NOSi}$ : C, 76.41; H, 7.53; N, 3.87. Found: C, 76.38; H, 7.36; N, 3. 64.

6e: N-((2-chlorophenyl)(thiophen-3-yl)methyl)-N-phenyl-O-(trimethylsilyl)hydroxylamine<smiles>CON(c1ccccc1)C(c1ccsc1)c1c(Cl)cccc1Cl</smiles>

According to general procedure A: A solution of TMSCl $(0.65 \mathrm{~g}, 0.78 \mathrm{ml}, 6.0 \mathrm{mmol})$ in THF $(5 \mathrm{~mL})$ was added slowly to a mixture of N-phenyl-C-(2-chlorophenyl) nitrone 2c (0.70 g, $3.0 \mathrm{mmol}), 3$ thienylzinc bromide $(4.5 \mathrm{mmol})$ in $10 \mathrm{ml}$ of THF under room temperature. The reaction mixture was stirred at this temperature for half an hour. The pure product was obtained as yellowish syrup $(0.88 \mathrm{~g}$, $2.28 \mathrm{mmol}, 76 \%)$ after purification on silica gel (50:1 petroleum ether : EtOAc).

IR $(\mathrm{KBr}) \vee\left(\mathrm{cm}^{-1}\right): 3452,2958,1592,1484,1251,895,842,754,700 ;{ }^{1} \mathrm{H} \mathrm{NMR}\left(400 \mathrm{MHz}, \mathrm{CDCl}_{3}\right) \delta$ (ppm): -0.24 (s, 9H), 6.38 (s, 1H), $6.84(\mathrm{dd}, J=5.2,3.6 \mathrm{~Hz}, 2 \mathrm{H}), 6.92(\mathrm{dd}, J=6.8,3.6 \mathrm{~Hz}, 1 \mathrm{H}), 7.12-$ $7.26(\mathrm{~m}, 5 \mathrm{H}), 7.29(\mathrm{dd}, J=8.8,1.2 \mathrm{~Hz}, 2 \mathrm{H}), 7.36-7.42(\mathrm{~m}, 1 \mathrm{H}), 7.64-7.70(\mathrm{~m}, 1 \mathrm{H}) .{ }^{13} \mathrm{C} \mathrm{NMR}(100 \mathrm{MHz}$, $\left.\mathrm{CDCl}_{3}\right) \delta(\mathrm{ppm}):-1.1,69.5,119.6,123.2,125.7,125.8,126.2,128.0,128.1,129.5,132.2,134.7,137.6$, 138.6, 152.4. MS (ESI, m/z): $387.8[\mathrm{M}+\mathrm{H}]^{+}$. Anal. Calcd for $\mathrm{C}_{20} \mathrm{H}_{22} \mathrm{ClNOSSi}: \mathrm{C}, 61.91 ; \mathrm{H}, 5.72 ; \mathrm{N}$, 3.61. Found: C, 61.77; H, 5.42; N, 3. 46.

6f: N-((4-fluorophenyl)(thiophen-3-yl)methyl)-N-phenyl-O-(trimethylsilyl)hydroxylamine

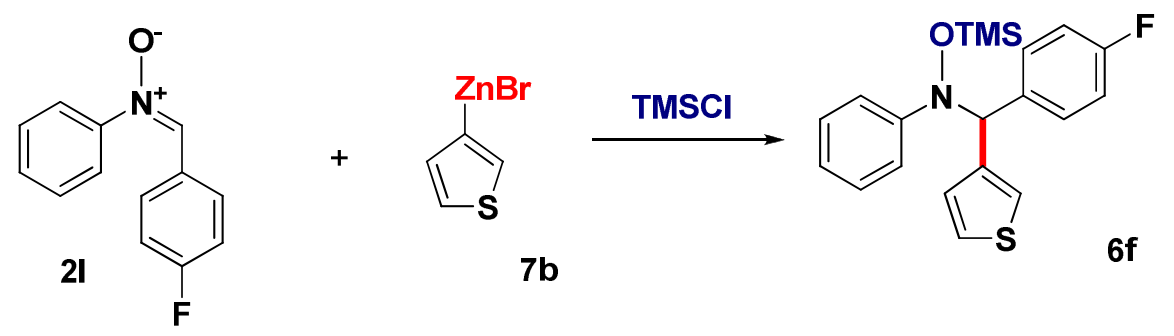

According to general procedure A: A solution of TMSCl $(0.65 \mathrm{~g}, 0.78 \mathrm{ml}, 6.0 \mathrm{mmol})$ in $\mathrm{THF}(5 \mathrm{~mL})$ was added slowly to a mixture of N-phenyl-C-(4-fluorophenyl) nitrone 21 (0.65 g, $3.0 \mathrm{mmol})$, 3thienylzinc bromide $(4.5 \mathrm{mmol})$ in $10 \mathrm{ml}$ of THF under room temperature. The reaction mixture was stirred at this temperature for half an hour. The pure product was obtained as yellowish syrup (0.86 $\mathrm{g}$, $2.31 \mathrm{mmol}, 77 \%)$ after purification on silica gel (50:1 petroleum ether : EtOAc).

IR $(\mathrm{KBr}) \vee\left(\mathrm{cm}^{-1}\right): 3447,2955,2924,1598,1510,1256,1231,844,758,690 ;{ }^{1} \mathrm{H}$ NMR (400 MHz, $\left.\mathrm{CDCl}_{3}\right) \delta(\mathrm{ppm}):-0.14(\mathrm{~s}, 9 \mathrm{H}), 5.71(\mathrm{~s}, 1 \mathrm{H}), 6.82(\mathrm{dd}, J=2.8,0.8 \mathrm{~Hz}, 2 \mathrm{H}), 6.86(\mathrm{dd}, J=5.2,3.6 \mathrm{~Hz}$, $1 \mathrm{H}), 6.92-7.02(\mathrm{~m}, 3 \mathrm{H}), 7.08-7.18(\mathrm{~m}, 4 \mathrm{H}), 7.22(\mathrm{dd}, J=5.2,0.8 \mathrm{~Hz}, 1 \mathrm{H}), 7.42(\mathrm{dd}, J=8.4,5.2 \mathrm{~Hz}$, $1 \mathrm{H}) ;{ }^{13} \mathrm{C} \mathrm{NMR}\left(100 \mathrm{MHz}, \mathrm{CDCl}_{3}\right) \delta(\mathrm{ppm}):-0.9,73.5,114.6(\mathrm{~d}, J=21 \mathrm{~Hz}, 2 \mathrm{C}), 120.4,123.7,125.8$, 
127.6, 128.1, 131.4, 131.5, 135.4, 140.6, 152.2, 162.2 (d, $J=245.0 \mathrm{~Hz}, 1 \mathrm{C}) ; \mathrm{MS}(\mathrm{ESI}, \mathrm{m} / \mathrm{z}): 371.8$ $[\mathrm{M}+\mathrm{H}]^{+}$; Anal. Calcd for $\mathrm{C}_{20} \mathrm{H}_{22}$ FNOSSi: C, 64.65; H, 5.97; N, 3.77. Found: C, 64.57; H, 5.76; N, 3.54.

6g: N-((2,4-dichlorophenyl)(thiophen-3-yl)methyl)-N-phenyl-O-(trimethylsilyl)hydroxylamine<smiles>CON(c1ccccc1)C(c1ccsc1)c1c(Cl)cc(Cl)cc1Cl</smiles>

According to general procedure A: A solution of TMSCl $(0.65 \mathrm{~g}, 0.78 \mathrm{ml}, 6.0 \mathrm{mmol})$ in THF $(5 \mathrm{~mL})$ was added slowly to a mixture of N-phenyl-C-(2,4-dichlorophenyl) nitrone $2 \mathbf{e}(0.80 \mathrm{~g}, 3.0 \mathrm{mmol}), 3$ thienylzinc bromide $(4.5 \mathrm{mmol})$ in $10 \mathrm{ml}$ of THF under room temperature. The reaction mixture was stirred at this temperature for half an hour. The pure product was obtained as colorless syrup (1.04 g, $2.46 \mathrm{mmol}, 82 \%)$ after purification on silica gel (50:1 petroleum ether : EtOAc).

IR $(\mathrm{KBr}) \vee\left(\mathrm{cm}^{-1}\right): 3442,2961,1637,1590,1485,1381,1252,843,748,693 ;{ }^{1} \mathrm{H}$ NMR (400 MHz, $\left.\mathrm{CDCl}_{3}\right) \delta(\mathrm{ppm}):-0.21(\mathrm{~s}, 9 \mathrm{H}), 6.30(\mathrm{~s}, 1 \mathrm{H}), 6.85(\mathrm{dd}, J=5.2,3.6 \mathrm{~Hz}, 2 \mathrm{H}), 6.89(\mathrm{~d}, J=2.8 \mathrm{~Hz}, 1 \mathrm{H})$, $6.94(\mathrm{t}, J=7.2 \mathrm{~Hz}, 1 \mathrm{H}), 7.15-7.23(\mathrm{~m}, 4 \mathrm{H}), 7.26(\mathrm{~d}, J=7.8 \mathrm{~Hz}, 1 \mathrm{H}), 7.40$ (d, $J=2.0 \mathrm{~Hz}, 1 \mathrm{H}), 7.63(\mathrm{~d}, J$ $=8.4 \mathrm{~Hz}, 1 \mathrm{H}) ;{ }^{13} \mathrm{C} \mathrm{NMR}\left(100 \mathrm{MHz}, \mathrm{CDCl}_{3}\right) \delta(\mathrm{ppm}):-1.0,69.0,119.7,123.5,125.9,126.1,126.5$, 128.1, 128.1, 129.2, 132.9, 133.9, 135.3, 136.2, 138.4, 152.1; MS (ESI, m/z): $421.7[\mathrm{M}+\mathrm{H}]^{+}$; Anal. Calcd for $\mathrm{C}_{20} \mathrm{H}_{21} \mathrm{Cl}_{2} \mathrm{NOSSi}$ : C, 56.86; H, 5.01; N, 3.32. Found: C, 56.64; H,4.88; N, 3.14.

\section{6h: N-(1-(4-methoxyphenyl)octyl)-N-phenyl-O-(trimethylsilyl)hydroxylamine}

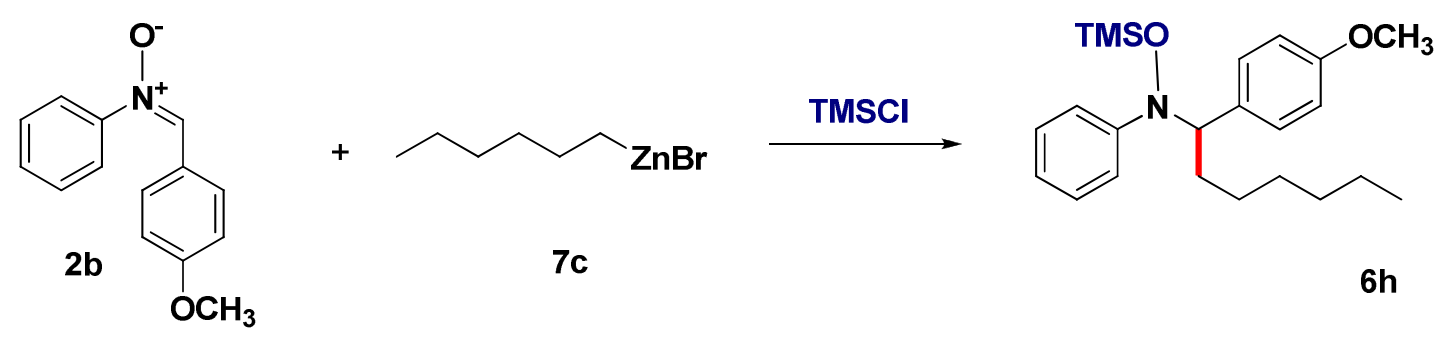

According to general procedure A: A solution of TMSCl $(0.65 \mathrm{~g}, 0.78 \mathrm{ml}, 6.0 \mathrm{mmol})$ in THF $(5 \mathrm{~mL})$ was added slowly to a mixture of N-phenyl-C-(4-methoxyphenyl) nitrone $2 \mathbf{b}$ (0.68 g, $3.0 \mathrm{mmol}), 1$ hexylzinc bromide $(4.5 \mathrm{mmol})$ in $10 \mathrm{ml}$ of THF under room temperature. The reaction mixture was stirred at this temperature for half an hour.The pure product was obtained as colorless oil $(0.84 \mathrm{~g}, 2.19$ mmol, 73\%) after purification on silica gel (50:1 petroleum ether : EtOAc). 
IR $(\mathrm{KBr}) \vee\left(\mathrm{cm}^{-1}\right): 2955,2928,2857,1608,1512,1485,1301,1249,1177,1035,877,842,759,698$; ${ }^{1} \mathrm{H}$ NMR $\left(400 \mathrm{MHz}, \mathrm{CDCl}_{3}\right) \delta(\mathrm{ppm}):-0.06(\mathrm{~s}, 9 \mathrm{H}), 0.83(\mathrm{t}, J=6.8 \mathrm{~Hz}, 3 \mathrm{H}), 1.10-1.30(\mathrm{~m}, 8 \mathrm{H}), 1.91(\mathrm{t}$, $J=8.0 \mathrm{~Hz}, 2 \mathrm{H}), 3.77(\mathrm{~s}, 3 \mathrm{H}), 4.08(\mathrm{t}, J=6.4 \mathrm{~Hz}, 1 \mathrm{H}), 6.76(\mathrm{dd}, J=8.8,2.4 \mathrm{~Hz}, 2 \mathrm{H}), 6.90-7.00(\mathrm{~m}, 3 \mathrm{H})$, $7.04(\mathrm{dd}, J=8.8,2.4 \mathrm{~Hz}, 2 \mathrm{H}), 7.10-7.20(\mathrm{~m}, 2 \mathrm{H}) ;{ }^{13} \mathrm{C} \mathrm{NMR}\left(100 \mathrm{MHz}, \mathrm{CDCl}_{3}\right) \delta$ (ppm): -0.5, 14.0, 22.6, 26.8, 29.3, 30.5, 31.7, 55.1, 73.8, 112.8, 120.7, 123.1, 127.8, 130.8, 131.1, 153.0, 158.6; MS (ESI, m/z): $385.9[\mathrm{M}+\mathrm{H}]^{+}$; Anal. Calcd for $\mathrm{C}_{23} \mathrm{H}_{35} \mathrm{NO}_{2} \mathrm{Si}: \mathrm{C}, 71.64 ; \mathrm{H}, 9.15 ; \mathrm{N}, 3.63$. Found: C, 71.46; H, $8.92 ; \mathrm{N}, 3.51$.

6i: N-phenyl-N-(1-(3,4,5-trimethoxyphenyl)octyl)-O-(trimethylsilyl)hydroxylamine

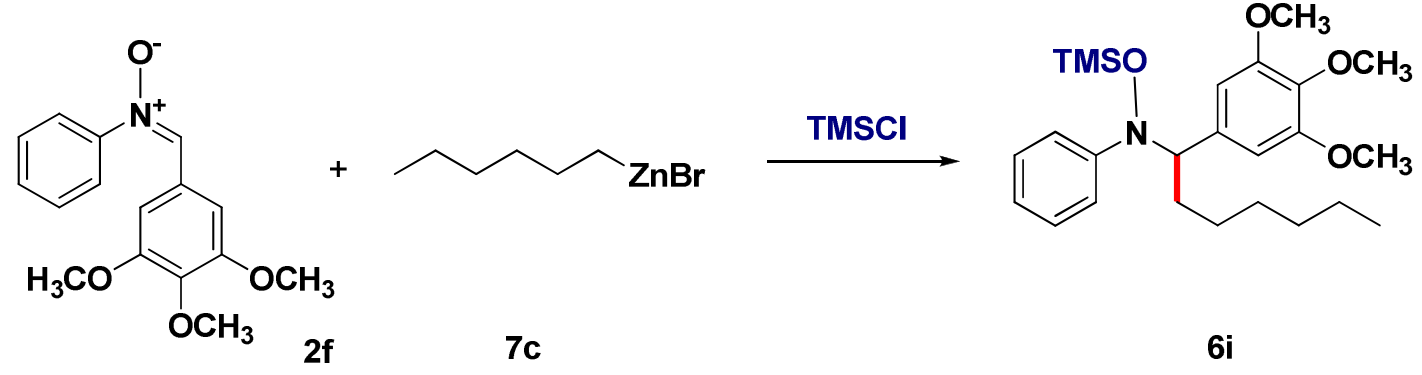

According to general procedure A: A solution of TMSCl $(0.65 \mathrm{~g}, 0.78 \mathrm{ml}, 6.0 \mathrm{mmol})$ in THF $(5 \mathrm{~mL})$ was added slowly to a mixture of N-phenyl-C-(3,4,5-trimethoxyphenyl) nitrone $2 \mathbf{f}(0.86 \mathrm{~g}, 3.0 \mathrm{mmol})$, 1-hexylzinc bromide $(4.5 \mathrm{mmol})$ in $10 \mathrm{ml}$ of THF under room temperature. The reaction mixture was stirred at this temperature for half an hour. The pure product was obtained as colorless oil (1.06 g, 2.37 mmol, 79\%) after purification on silica gel (50:1 petroleum ether : EtOAc).

IR $(\mathrm{KBr}) \vee\left(\mathrm{cm}^{-1}\right): 2953,2931,2857,1591,1506,1460,1331,1244,1130,1021,878,843,763,698$; ${ }^{1} \mathrm{H}$ NMR (400 MHz, $\left.\mathrm{CDCl}_{3}\right) \delta(\mathrm{ppm}):-0.46(\mathrm{~s}, 9 \mathrm{H}), 0.84(\mathrm{t}, J=7.2 \mathrm{~Hz}, 3 \mathrm{H}), 1.10-1.30(\mathrm{~m}, 8 \mathrm{H}), 1.80-$ $1.95(\mathrm{~m}, 2 \mathrm{H}), 3.74(\mathrm{~s}, 6 \mathrm{H}), 3.82(\mathrm{~s}, 3 \mathrm{H}), 4.03(\mathrm{dd}, J=8.8,6.0 \mathrm{~Hz}, 1 \mathrm{H}), 6.31(\mathrm{~s}, 2 \mathrm{H}), 6.92-7.03(\mathrm{~m}, 3 \mathrm{H})$, $7.17(\mathrm{t}, J=8.4 \mathrm{~Hz}, 2 \mathrm{H}) ;{ }^{13} \mathrm{C} \mathrm{NMR}\left(100 \mathrm{MHz}, \mathrm{CDCl}_{3}\right) \delta(\mathrm{ppm}):-0.5,14.0,22.6,26.8,29.3,30.6,31.7$, 56.0, 60.8, 74.7, 106.9, 120.8, 123.3, 127.8, 134.3, 137.2, 152.2, 152.8; MS (ESI, m/z):445.1 [M] $]^{+}$; Anal. Calcd for $\mathrm{C}_{25} \mathrm{H}_{39} \mathrm{NO}_{4} \mathrm{Si}$ : C, 67.37; H, 8.82; N, 3.14. Found: C, 67.24; H, 8.65; N, 2.86.

6j: N-phenyl-N-(1-(thiophen-3-yl)octyl)-O-(trimethylsilyl)hydroxylamine

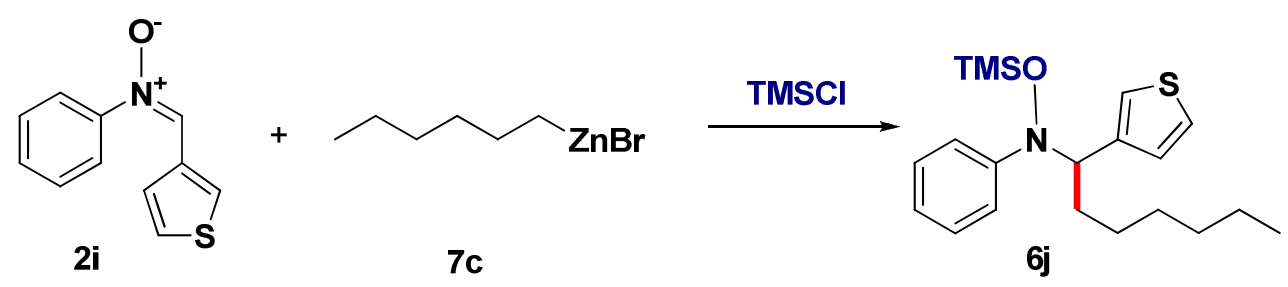

According to general procedure A: A solution of TMSCl $(0.65 \mathrm{~g}, 0.78 \mathrm{ml}, 6.0 \mathrm{mmol})$ in THF $(5 \mathrm{~mL})$ was added slowly to a mixture of N-phenyl-C-(3-thienyl) nitrone $2 \mathbf{i}(0.61 \mathrm{~g}, 3.0 \mathrm{mmol})$, 1-hexylzinc 
bromide $(4.5 \mathrm{mmol})$ in $10 \mathrm{ml}$ of THF under room temperature. The reaction mixture was stirred at this temperature for half an hour. The pure product was obtained as yellowish oil $(0.80 \mathrm{~g}, 2.22 \mathrm{mmol}, 74 \%)$ after purification on silica gel (50:1 petroleum ether : EtOAc).

IR $(\mathrm{KBr}) \vee\left(\mathrm{cm}^{-1}\right): 3435,2954,2926,2856,1595,1486,1454,1308,1250,922,876,843,760,696 ;{ }^{1} \mathrm{H}$ NMR (400 MHz, $\left.\mathrm{CDCl}_{3}\right) \delta(\mathrm{ppm}): 0.02(\mathrm{~s}, 9 \mathrm{H}) .0 .85$ (t, $\left.J=6.8 \mathrm{~Hz}, 3 \mathrm{H}\right), 1.15-1.35(\mathrm{~m}, 8 \mathrm{H}), 1.80-2.06$ $(\mathrm{m}, 2 \mathrm{H}), 4.41(\mathrm{dd}, J=9.2,5.2 \mathrm{~Hz}, 1 \mathrm{H}), 6.61(\mathrm{~d}, J=2.4 \mathrm{~Hz}, 1 \mathrm{H}), 6.83(\mathrm{dd}, J=5.1,3.5 \mathrm{~Hz}, 1 \mathrm{H}), 6.92-$ 7.01 (m, 3H), 7.13-7.20 (m, 3H); ${ }^{13} \mathrm{C}$ NMR (100MHz, $\left.\mathrm{CDCl}_{3}\right) \delta$ (ppm): -0.5, 14.0, 22.6, 26.9, 29.1, 31.7, 70.1, 120.2, 123.2, 124.7, 125.5, 126.4, 127.9, 152.6; MS (ESI, m/z): $362.0[\mathrm{M}+\mathrm{H}]^{+}$; Anal. Calcd for $\mathrm{C}_{20} \mathrm{H}_{31} \mathrm{NOSSi}$ : C, 66.43; H, 8.64; N, 3.87. Found: C, 66.23; H, 8.46; N, 3.76.

6k: (R)-N-(1-(2-chlorophenyl)octyl)-N-phenyl-O-(trimethylsilyl)hydroxylamine
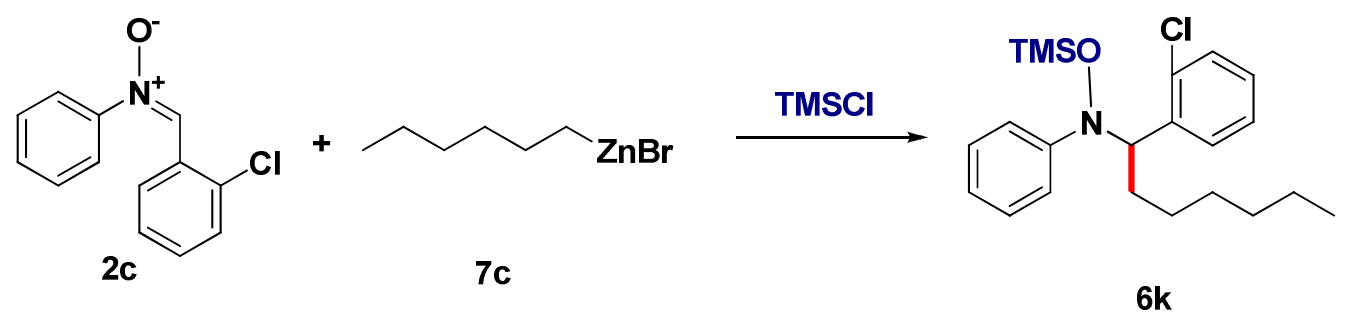

According to general procedure A: A solution of TMSCl $(0.65 \mathrm{~g}, 0.78 \mathrm{ml}, 6.0 \mathrm{mmol})$ in THF $(5 \mathrm{~mL})$ was added slowly to a mixture of N-phenyl-C-(2-chlorophenyl) nitrone 2c (0.70 g, $3.0 \mathrm{mmol})$, 1hexylzinc bromide $(4.5 \mathrm{mmol})$ in $10 \mathrm{ml}$ of THF under room temperature. The reaction mixture was stirred at this temperature for half an hour. The pure product was obtained as yellowish oil $(0.77 \mathrm{~g}, 1.98$ mmol, 66\%) after purification on silica gel (50:1 petroleum ether : EtOAc).

IR $(\mathrm{KBr}) \vee\left(\mathrm{cm}^{-1}\right): 2954,2925,2856,1595,1485,1446,1250,1036,904,877,843,754,697 ;{ }^{1} \mathrm{H} \mathrm{NMR}$ $\left(400 \mathrm{MHz}, \mathrm{CDCl}_{3}\right) \delta(\mathrm{ppm}):-0.23(\mathrm{~s}, 9 \mathrm{H}), 0.88(\mathrm{t}, J=7.2 \mathrm{~Hz}, 3 \mathrm{H}), .95-1.22(\mathrm{~m}, 8 \mathrm{H}), 1.76-1.83$

(m, 1H), 2.00-2.13 (m, 1H), $4.84(\mathrm{dd}, J=10.8,4.4 \mathrm{~Hz}, 1 \mathrm{H}), 6.92-7.03(\mathrm{~m}, 1 \mathrm{H}), 7.15-7.28(\mathrm{~m}, 6 \mathrm{H})$, $7.34(\mathrm{dd}, J=7.6,1.2 \mathrm{~Hz}, 1 \mathrm{H}), 7.48(\mathrm{dd}, \mathrm{J}=7.6,1.2 \mathrm{~Hz}, 1 \mathrm{H}) ;{ }^{13} \mathrm{C} \mathrm{NMR}\left(100 \mathrm{MHz}, \mathrm{CDCl}_{3}\right) \delta(\mathrm{ppm})$ : 0.9, 14.0, 22.5, 26.3, 29.1, 29.7, 31.6, 68.8, 119.9, 122.9, 126.0, 128.1, 128.4, 129.3, 130.6, 136.1, 136.9, 152.9; MS (ESI, m/z):390.2 [M+H] $]^{+}$; Anal. Calcd for $\mathrm{C}_{22} \mathrm{H}_{32}$ ClNOSi: C, 67.75; H, 8.27; N, 3.59. Found: C, 67.46; H, 8.09; N, 3.64.

\section{6l: N-((2-methyl-1-(p-tolyl)butyl)-N-phenyl-O-(trimethylsilyl)hydroxylamine}<smiles>Cc1ccc(/C=[N+](/[O-])c2ccccc2)cc1</smiles>

$2 \mathrm{~h}$

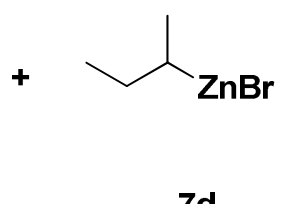

$7 d$

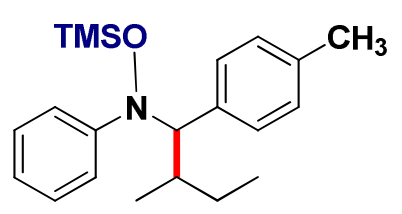

61 
According to general procedure A: A solution of TMSCl $(0.65 \mathrm{~g}, 0.78 \mathrm{ml}, 6.0 \mathrm{mmol})$ in THF $(5 \mathrm{~mL})$ was added slowly to a mixture of N-(4-methylbenzylidene)aniline oxide $\mathbf{2 h}(0.63 \mathrm{~g}, 3.0 \mathrm{mmol})$, 2butylzinc bromide $(4.5 \mathrm{mmol})$ in $10 \mathrm{ml}$ of THF under room temperature. The reaction mixture was stirred at this temperature for half an hour. The pure product was obtained as yellowish oil $(0.55 \mathrm{~g}, 1.62$ mmol, 54\%) after purification on silica gel (50:1 petroleum ether : EtOAc).

IR $(\mathrm{KBr}) \vee\left(\mathrm{cm}^{-1}\right): 3394,2961,2924,2873,1595,1485,1451,1250,922,887,843,758,696 ;{ }^{1} \mathrm{H}$ NMR $\left(400 \mathrm{MHz}, \mathrm{CDCl}_{3}\right) \delta(\mathrm{ppm}): 0.06$ (s, 9H, $\left.\mathrm{Si}\left(\mathrm{CH}_{3}\right)_{3}\right), 0.07$ (s, 9H, $\left.\mathrm{Si}\left(\mathrm{CH}_{3}\right)_{3}\right), 0.64(\mathrm{~d}, J=6.8 \mathrm{~Hz}, 3 \mathrm{H}$, $\left.\mathrm{CH}_{3}\right), 0.77\left(\mathrm{t}, J=6.4 \mathrm{~Hz}, 3 \mathrm{H}, \mathrm{CH}_{3}\right), 0.80-0.92\left(\mathrm{~m}, 2 \mathrm{H}, \mathrm{CH}_{2}\right), 1.04\left(\mathrm{t}, J=7.2 \mathrm{~Hz}, 3 \mathrm{H}, \mathrm{CH}_{3}\right), 1.20-1.30$ (m, 2H, $\mathrm{CH}_{2}$ ), 1.34 (d, J = 6.4 Hz, 3H, $\mathrm{CH}_{3}$ ), 2.05-2.20 (m, 1H, CH), $2.27\left(\mathrm{~s}, 6 \mathrm{H}, 2 \times \mathrm{CH}_{3}\right), 2.30-2.40$ $(\mathrm{m}, 1 \mathrm{H}, \mathrm{CH}), 3.72(\mathrm{~d}, J=2.0 \mathrm{~Hz}, 1 \mathrm{H}, \mathrm{CH}), 3.74(\mathrm{~d}, J=2.0,1 \mathrm{H}, \mathrm{CH}), 6.75(\mathrm{dd}, J=8.0,2.8 \mathrm{~Hz}, 4 \mathrm{H}$, $\mathrm{ArH}), 6.85(\mathrm{dd}, J=7.6,2.0 \mathrm{~Hz}, 4 \mathrm{H}, \mathrm{ArH}), 6.88-6.96(\mathrm{~m}, 6 \mathrm{H}, \mathrm{ArH}), 7.18(\mathrm{t}, J=7.6 \mathrm{~Hz}, 4 \mathrm{H}, \mathrm{ArH}) ;{ }^{13} \mathrm{C}$ NMR $\left(100 \mathrm{MHz}, \mathrm{CDCl}_{3}\right) \delta(\mathrm{ppm}):-0.1,0.0,10.7,11.2,16.5,17.6,26.4,27.1,35.2,35.3,80.5,80.7$, 120.2 , 120.4, 122.7, 122.4, 127.4, 127.5, 130.3, 130.4, 132.9, 133.1, 136.2, 136.2, 153.5, 153.6; MS (ESI, m/z):342.1 [M] ${ }^{+}$; Anal. Calcd for $\mathrm{C}_{21} \mathrm{H}_{31} \mathrm{NOSi}$ : C, 73.84; H, 9.15; N, 4.10. Found: C, 73.69; $\mathrm{H}$, $9.06 ; \mathrm{N}, 3.89$.

6m: N-((4-methoxyphenyl)-2-methylbutyl)-N-phenyl-O-(trimethylsilyl)hydroxylamine<smiles>COc1ccc(/C=[N+](\[O-])c2ccccc2)cc1</smiles>

2b

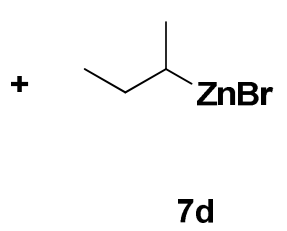

$7 d$

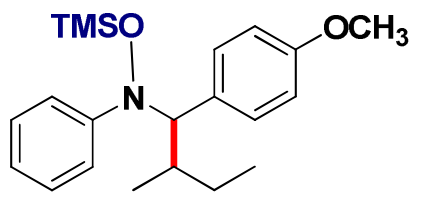

$6 m$

According to general procedure A: A solution of TMSCl $(0.65 \mathrm{~g}, 0.78 \mathrm{ml}, 6.0 \mathrm{mmol})$ in THF $(5 \mathrm{~mL})$ was added slowly to a mixture of N-phenyl-C-(4-methoxyphenyl) nitrone $\mathbf{2 b}$ (0.68 g, $3.0 \mathrm{mmol})$, 2butylzinc bromide $(4.5 \mathrm{mmol})$ in $10 \mathrm{ml}$ of THF under room temperature. The reaction mixture was stirred at this temperature for half an hour. The pure product was obtained as yellowish oil (0.58 g, 1.62 mmol, 54\%) after purification on silica gel (30:1 petroleum ether : EtOAc).

IR $(\mathrm{KBr}) \vee\left(\mathrm{cm}^{-1}\right): 3375,2958,2924,2854,1597,1488,1462,1246,1032,922,888,842,753,696 ;{ }^{1} \mathrm{H}$ NMR (400 MHz, $\left.\mathrm{CDCl}_{3}\right) \delta(\mathrm{ppm}):-0.04$ (s, 9H, Si( $\left.\left.\mathrm{CH}_{3}\right)_{3}\right),-0.08$ (s, 9H, $\left.\mathrm{Si}\left(\mathrm{CH}_{3}\right)_{3}\right), 0.64$ (d, J = 6.8 Hz, $\left.3 \mathrm{H}, \mathrm{CH}_{3}\right), 0.76\left(\mathrm{t}, J=6.4 \mathrm{~Hz}, 3 \mathrm{H}, \mathrm{CH}_{3}\right), 0.82-0.92\left(\mathrm{~m}, 2 \mathrm{H}, \mathrm{CH}_{2}\right), 1.04\left(\mathrm{t}, J=7.2 \mathrm{~Hz}, 3 \mathrm{H}, \mathrm{CH}_{3}\right), 1.20-$ $1.30\left(\mathrm{~m}, 2 \mathrm{H}, \mathrm{CH}_{2}\right), 1.34$ (d, J = 6.4 Hz, 3H, $\left.\mathrm{CH}_{3}\right), 2.05-2.44(\mathrm{~m}, 2 \mathrm{H}, \mathrm{CH}), 3.20$ (s, 3H, $\left.\mathrm{OCH}_{3}\right), 3.22(\mathrm{~s}$, $\left.3 \mathrm{H}, \mathrm{OCH}_{3}\right), 4.51(\mathrm{~d}, J=4.0 \mathrm{~Hz}, 1 \mathrm{H}, \mathrm{CH}), 4.54(\mathrm{~d}, J=4.0,1 \mathrm{H}, \mathrm{CH}), 6.60-6,68(\mathrm{~m}, 4 \mathrm{H}, \mathrm{ArH}), 6.80-6.95$ $(\mathrm{m}, 8 \mathrm{H}, \mathrm{ArH}), 7.05-7.18$ (m, 6H, ArH); $\left.{ }^{13} \mathrm{C} \mathrm{NMR} \mathrm{(100MHz,} \mathrm{CDCl}_{3}\right) \delta(\mathrm{ppm}):-0.1,-0.1,10.6,11.1$, $16.1,17.8,26.2,29.7,34.7,34.9,55.3,110.4,119.1,119.7,120.0,122.2,122.3,127.4,127.4$, 131.4, 
131.6, 154.3, 157.9; MS (ESI, m/z): 358.1, [M+H] ${ }^{+}$; Anal. Calcd for $\mathrm{C}_{21} \mathrm{H}_{31} \mathrm{NO}_{2} \mathrm{Si}$ : C,70.54; H, 8.74; N, 3.92. Found: C, 70.89; H, 8.68; N, 3.64.

6n: N-(naphthalen-1-yl(p-tolyl)methyl)-N-phenyl-O-(trimethylsilyl)hydroxylamine

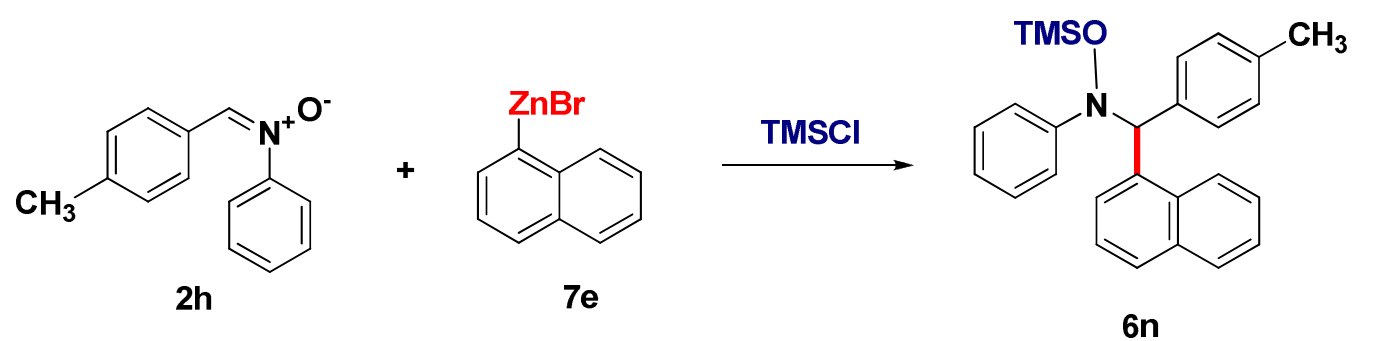

According to general procedure A: A solution of $\mathrm{TMSCl}(0.65 \mathrm{~g}, 0.78 \mathrm{ml}, 6.0 \mathrm{mmol})$ in THF (5 mL) was added slowly to a mixture of N-phenyl-C-(4-methylphenyl) nitrone $\mathbf{2 h}$ (0.63 g, $3.0 \mathrm{mmol})$, 1naphthylzinc bromide $(4.5 \mathrm{mmol})$ in $10 \mathrm{ml}$ of THF under room temperature. The reaction mixture was stirred at this temperature for half an hour. The pure product was obtained as yellowish oil $(0.90 \mathrm{~g}, 2.19$ mmol, 73\%) after purification on silica gel (30:1 petroleum ether : EtOAc).

IR $(\mathrm{KBr}) \vee\left(\mathrm{cm}^{-1}\right): 3401,2925,1595,1510,1487,1451,1330,1250,1130,916,897,843,779,752,694$. ${ }^{1} \mathrm{H}$ NMR $\left(400 \mathrm{MHz}, \mathrm{CDCl}_{3}\right) \delta(\mathrm{ppm}):-0.35(\mathrm{~s}, 9 \mathrm{H}), 2.26(\mathrm{~s}, 3 \mathrm{H}), 6.50(\mathrm{~s}, 1 \mathrm{H}), 6.85(\mathrm{tt}, J=7.2,0.9 \mathrm{~Hz}$, $1 \mathrm{H}), 7.01(\mathrm{~d}, J=8.0 \mathrm{~Hz}, 2 \mathrm{H}), 7.15(\mathrm{tt}, J=7.2,0.9 \mathrm{~Hz}, 2 \mathrm{H}), 7.26(\mathrm{dd}, J=8.8,0.9 \mathrm{~Hz}, 2 \mathrm{H}), 7.34-7.50(\mathrm{~m}$, $5 \mathrm{H}), 7.72(\mathrm{t}, J=8.0 \mathrm{~Hz}, 2 \mathrm{H}), 7.81(\mathrm{~d}, J=8.0,1 \mathrm{H}), 8.26(\mathrm{~d}, J=8.0 \mathrm{~Hz}, 1 \mathrm{H}) ;{ }^{13} \mathrm{C} \mathrm{NMR}(100 \mathrm{MHz}$, $\left.\mathrm{CDCl}_{3}\right) \delta(\mathrm{ppm}):-0.7,21.1,29.7,71.8,119.1,122.4,123.7,125.0,125.9,127.8,128.2,128.5,128.6$, 128.7, 130.2, 132.8, 136.2, 136.5, 136.6, 153.1; MS (ESI, m/z): $411.9[\mathrm{M}]^{+}$; Anal. Calcd for $\mathrm{C}_{27} \mathrm{H}_{29}$ NOSi: C, 78.79; H, 7.10; N, 3.89. Found: C, 78.64; H, 6.84; N, 3.26.

\section{General procedure B: Reduction of O-trimethylsilyl hydroxylamines into amine.}

The O-trimethylsilyl hydroxylamine $(2 \mathrm{mmol})$ was dissolved into a 2:1 solution of EtOH and sat. aq. $\mathrm{NH}_{4} \mathrm{Cl}(20 \mathrm{~mL})$ in a $50 \mathrm{~mL}$ round bottomed flask. $\mathrm{Cu}(\mathrm{OAc})_{2}$ powder $(40 \mathrm{mg}, 0.2 \mathrm{mmol})$ and $\mathrm{Zn}(260$ $\mathrm{mg}, 4 \mathrm{mmol}$ ) were added and the mixture is stirred and heated under nitrogen atmosphere at $50{ }^{\circ} \mathrm{C}$ for several hours until the hydroxylime was completely consumed (TLC control). The mixture was cooled, filtered over Celite and concentrated. Then, a sat. $\mathrm{Na}_{2} \mathrm{CO}_{3}$ solution $(15 \mathrm{~mL})$ was added and the product is extracted with ethyl acetate $(3 \times 15 \mathrm{~mL})$. The organic phase is dried over anhydrous $\mathrm{Na}_{2} \mathrm{SO}_{4}$, then filtered and concentrated to afford the desired amine, which was then purified by flash column chromatography.

\section{8a: N-(1-(4-methoxyphenyl)-2-phenylethyl)aniline}


<smiles>COc1ccc(C(Cc2ccccc2)Nc2ccccc2)cc1</smiles>

According to general procedure B: The O-trimethylsilyl hydroxylamine $5 \mathbf{b}(0.78 \mathrm{~g}, 2 \mathrm{mmol})$ was dissolved into a 2:1 solution of $\mathrm{EtOH}$ and sat. aq. $\mathrm{NH}_{4} \mathrm{Cl}(20 \mathrm{~mL})$ in a $50 \mathrm{~mL}$ round bottomed flask. $\mathrm{Cu}(\mathrm{OAc})_{2}$ powder $(40 \mathrm{mg}, 0.2 \mathrm{mmol})$ and $\mathrm{Zn}(260 \mathrm{mg}, 4 \mathrm{mmol})$ were added and the mixture is stirred and heated under nitrogen atmosphere at room temperature for 2 hours. The pure product was obtained as yellowish syrup (0.46 g, $1.54 \mathrm{mmol}, 77 \%)$ after purification on silica gel (30:1 petroleum ether : EtOAc).

IR $(\mathrm{KBr}) \vee\left(\mathrm{cm}^{-1}\right): 3409,2953,2919,2850,1602,1507,1245,1223 ;{ }^{1} \mathrm{H}$ NMR $\left(400 \mathrm{MHz}, \mathrm{CDCl}_{3}\right) \delta$ (ppm): 3.30 (dd, $J=14.0,8.0 \mathrm{~Hz}, 1 \mathrm{H}), 3.10(\mathrm{dd}, J=14.0,5.6 \mathrm{hz}, 1 \mathrm{H}), 3.7$ (s, 3H), 4.09 (s, 1H), 4.54 (t, $J=6.8 \mathrm{~Hz}, 1 \mathrm{H}), 6.46(\mathrm{~d}, J=8.4 \mathrm{~Hz}, 2 \mathrm{H}), 6.62(\mathrm{td}, J=7.6,0.9 \mathrm{~Hz}, 1 \mathrm{H}), 6.83(\mathrm{~d}, J=8.4 \mathrm{~Hz}, 2 \mathrm{H}), 7.05(\mathrm{t}$, $J=7.2 \mathrm{~Hz}, 2 \mathrm{H}), 7.10(\mathrm{~d}, J=7.6 \mathrm{~Hz}, 2 \mathrm{H}), 7.15-7.30(\mathrm{~m}, 5 \mathrm{H}) .{ }^{13} \mathrm{C} \mathrm{NMR}\left(100 \mathrm{MHz}, \mathrm{CDCl}_{3}\right) \delta(\mathrm{ppm})$ : 29.7, 45.1, 55.1, 58.6, 113.6, 113.8, 117.4, 126.6, 127.4, 128.4, 128.9, 129.2, 135.2, 137.7, 147.2, 158.5. HR-MS (ESI) calcd for C21H22NO [M+H] $]^{+}$304.4055; found 304.4056.

\section{8b: N-(2-phenyl-1-(3,4,5-trimethoxyphenyl)ethyl)aniline}

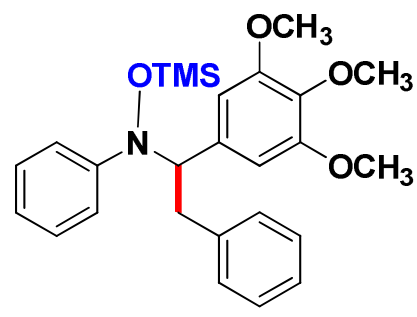

$5 f$

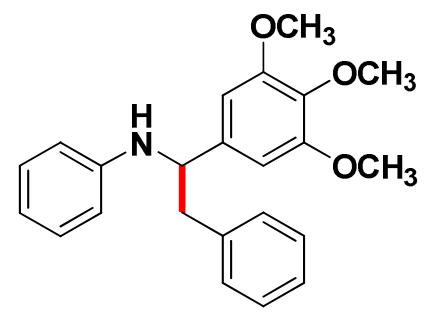

$8 b$

According to general procedure B: The O-trimethylsilyl hydroxylamine $\mathbf{5 f}(0.90 \mathrm{~g}, 2 \mathrm{mmol})$ was dissolved into a 2:1 solution of $\mathrm{EtOH}$ and sat. aq. $\mathrm{NH}_{4} \mathrm{Cl}(20 \mathrm{~mL})$ in a $50 \mathrm{~mL}$ round bottomed flask. $\mathrm{Cu}(\mathrm{OAc})_{2}$ powder $(40 \mathrm{mg}, 0.2 \mathrm{mmol})$ and $\mathrm{Zn}(260 \mathrm{mg}, 4 \mathrm{mmol})$ were added and the mixture is stirred and heated under nitrogen atmosphere at room temperature for 2 hours. The pure product was obtained as yellowish syrup (0.52 g, $1.44 \mathrm{mmol}, 72 \%)$ after purification on silica gel (20:1 petroleum ether : EtOAc). 
IR $(\mathrm{KBr}) \vee\left(\mathrm{cm}^{-1}\right): 3391,3057,2924,1599,1504,1234,1126,1028,1008,981 ;{ }^{1} \mathrm{H}$ NMR (400 MHz, $\left.\mathrm{CDCl}_{3}\right), \delta(\mathrm{ppm}): 3.03(\mathrm{dd}, J=13.7,10.5 \mathrm{~Hz}, 1 \mathrm{H}), 3.09$ (dd, $\left.J=13.9,5.9 \mathrm{~Hz}, 1 \mathrm{H}\right), 3.78(\mathrm{~s}, 6 \mathrm{H}), 3.83(\mathrm{~s}$, $3 \mathrm{H}), 4.09(\mathrm{~s}, 1 \mathrm{H}), 4.56(\mathrm{t}, J=13.9, \mathrm{~Hz}, 1 \mathrm{H}), 6.46-6.40(\mathrm{~m}, 2 \mathrm{H}), 6.51(\mathrm{~s}, 2 \mathrm{H}), 7.05-7.14(\mathrm{~m}, 4 \mathrm{H}), 7.21-$ $7.30(\mathrm{~m}, 4 \mathrm{H}) ;{ }^{13} \mathrm{C} \mathrm{NMR}\left(100 \mathrm{MHz}, \mathrm{CDCl}_{3}\right), \delta(\mathrm{ppm}): 45.2,56.0,59.8,65.3,103.1,113.7,126.7,126.9$, 127.6, 128.6, 129,0, 129.2, 137.5, 139.2, 147.3, 153.2. HR-MS (ESI) calcd for $\mathrm{C}_{23} \mathrm{H}_{26} \mathrm{NO}_{3}:[\mathrm{M}+\mathrm{H}]^{+}$: 364.1913; found 364.1914.

10. (2R, 3R, 4S, 5S)- N-trimethylsiloxy-2-vinyl-3,4-O-isopropylidene-5-[(R)-2,2-dimethyl-1,3dioxolan-4-yl]pyrrolidine.
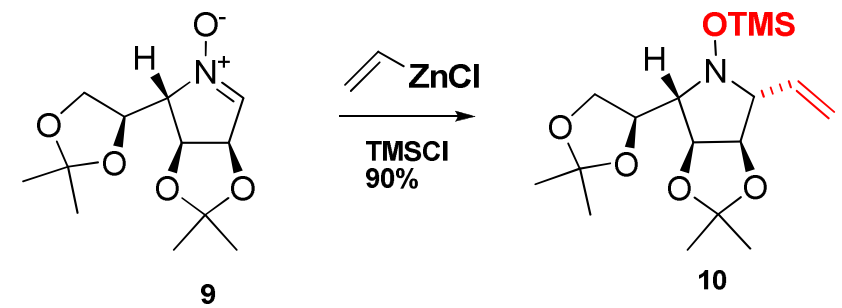

According to general procedure A: A solution of TMSCl $(0.73 \mathrm{~g}, 0.88 \mathrm{ml}, 6.76 \mathrm{mmol})$ in THF (5 mL) was added slowly to a mixture of nitrone 9 Nitrone $(0.87 \mathrm{~g}, 3.38 \mathrm{mmol})$ was dissolved in $20 \mathrm{ml}$ THF and the resulting mixture was cooled in a ice-water bath under argone. A solution of zinc chloride $(0.70$ $\mathrm{g}, 5.15 \mathrm{mmol})$ in THF $(10 \mathrm{ml})$ was vinylzinc bromide $(1.6 \mathrm{M}, 3.17 \mathrm{ml}, 5.07 \mathrm{mmol}, 1.5 \mathrm{eq}$.) was added dropwise. The reaction mioxture was stirred 1 hour at room temperature, TLC showed that all the starting material was consumed. Saturated ammonium chloride $(20 \mathrm{ml})$ was added, the resulting mixture was extracted with ethyl acetate $(3 \times 20 \mathrm{ml})$. the organic mixture was dried $\left(\mathrm{Na}_{2} \mathrm{SO}_{4}\right)$, and the solvent was evaporated to give a syrup which was chromatography to give the titled compound (1.01 g, $84 \%)$

$[\alpha]_{\mathrm{D}}^{20}=+7.10 .\left(\mathrm{c}=0.87, \mathrm{CHCl}_{3}\right), \mathrm{IR} v\left(\mathrm{~cm}^{-1}\right): 2987,2937,1647,1381,1258,1212,1071 .{ }^{1} \mathrm{H} \mathrm{NMR}$ $\left(\mathrm{CDCl}_{3}\right) \delta(\mathrm{ppm}):-0.04(9 \mathrm{H}, \mathrm{TMS}), 1.22,1.29,1.41,1.47\left(4 \times \mathrm{s}, 12 \mathrm{H}, \mathrm{CH}_{3}\right), 3.06(\mathrm{dd}, J=6.2,4.6 \mathrm{~Hz}$, 1H, H-5), 3.34 (dd, $J=6.3,6.3 \mathrm{~Hz}, 1 \mathrm{H}, \mathrm{H}-2), 3.90$ (dd, $J=7.8,5.1 \mathrm{~Hz}, 1 \mathrm{H}, \mathrm{H}-4$ ') 4.04 (dd, J = 9.2, 6.4 Hz, 1H, H-4'), 4.16-4.28 (m, 2H, H-3 and H-4), 4.33 (dd, $J=12.3,7.2$ Hz, 1H, H-5'), 5.22 (d, J = 10.2 $\left.\mathrm{Hz}, 1 \mathrm{H}, \mathrm{CH}_{2}=\mathrm{CH}\right), 5.34\left(\mathrm{~d}, J=17.1 \mathrm{~Hz}, 1 \mathrm{H}, \mathrm{CH}_{2}=\mathrm{CH}\right), 5.80-5.94\left(\mathrm{~m}, 1 \mathrm{H}, \mathrm{CH}_{2}=\mathrm{CH}\right) .{ }^{13} \mathrm{C} \mathrm{NMR}$ $\left(\mathrm{CDCl}_{3}\right) \delta(\mathrm{ppm}):-1.08,25.13\left(\mathrm{CH}_{3}\right), 25.18\left(\mathrm{CH}_{3}\right), 26.46\left(\mathrm{CH}_{3}\right), 27.21\left(\mathrm{CH}_{3}\right), 66.18(\mathrm{C}-4), 74.19(\mathrm{C}-$ 5'), 75.91 (C-2), 76.35 (C-5), 77.43 (C-4), 80.31 (C-3), $109.87\left(\mathrm{Me}_{2} \mathrm{CO}\right), 113.80\left(\mathrm{Me}_{2} \mathrm{CO}\right), 118.98$ $\left(\mathrm{CH}_{2}=\mathrm{CH}\right), 136.00\left(\mathrm{CH}_{2}=\mathrm{CH}\right)$. HR-MS (ESI): Calcd for $\mathrm{C}_{17} \mathrm{H}_{32} \mathrm{NO}_{5} \mathrm{Si}[\mathrm{M}+\mathrm{H}]^{+}:$358.2050; found 358.2053.

11. (2R, 3R, 4S, 5S)-2-vinyl-3,4-O-isopropylidene-5-[(R)-2,2-dimethyl-1,3-dioxolan-4yl]pyrrolidine 

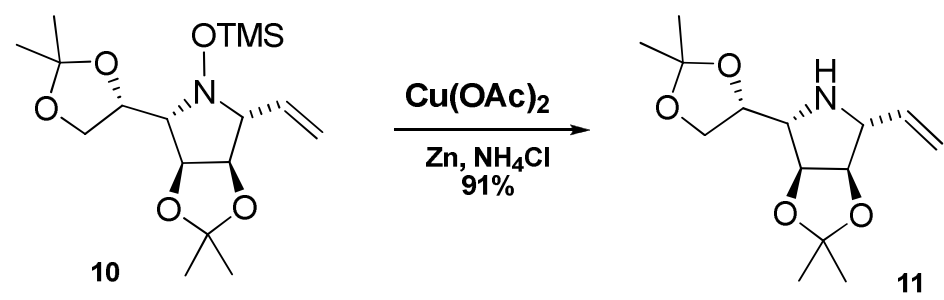

Compound 10 (1.01g, $2.82 \mathrm{mmol})$ was disolved in a solution of $20 \mathrm{ml}$ of dichloromethane. $20 \mathrm{ml}$ of saturated $\mathrm{NH}_{4} \mathrm{Cl}$ solution, zinc powder $(1.2 \mathrm{~g}, 18 \mathrm{mmol})$ and $\mathrm{Cu}(\mathrm{OAc})_{2}(360 \mathrm{mg}, 1.8 \mathrm{mmol})$ were then added and the mixture was stirred at room temperature for 2 hours. TLC showed that all the starting material was exhausted. The organic solution was separated and the aqueous phase was extracted with dichloromethane $(20 \mathrm{~mL})$. The organic phases were collected and washed with brine, dried $\left(\mathrm{MgSO}_{4}\right)$ and concentrated. The pure product $(0.69 \mathrm{~g}, 91 \%)$ was obtained after column chromatography using ethyl acetate/petroleum ether $(5 / 1, \mathrm{v} / \mathrm{v})$ as the eluant. $\mathrm{R}_{f}=0.47$ (ethyl acetate/petroleum ether $\left.1 / 4\right)$. $[\alpha]_{\mathrm{D}}^{20}$ $=+4.1 .\left(\mathrm{c}=2.4, \mathrm{CHCl}_{3}\right), \mathrm{IR} v\left(\mathrm{~cm}^{-1}\right): 3339, \quad 2986, \quad 2936, \quad 1645, \quad 1385, \quad 1375, \quad 1210,1071 。{ }^{1} \mathrm{H}$ NMR $\left(\mathrm{CDCl}_{3}\right) \delta(\mathrm{ppm}): 1.29(\mathrm{~s}, 3 \mathrm{H}), 1.37$ (s, 3H), 1.49 (s, 3H), 1.54 (s, 3H), 2.51 (brs, 1H,), 3.14 (dd, J $=6.3,4.8 \mathrm{~Hz}, 1 \mathrm{H}), 3.42(\mathrm{t}, J=6.6 \mathrm{~Hz}, 1 \mathrm{H}), 3.94(\mathrm{dd}, J=7.8,4.5 \mathrm{~Hz}, 1 \mathrm{H}), 4.09(\mathrm{dd}, J=9.0,6.9 \mathrm{~Hz}$, $1 \mathrm{H}), 4.19-4.29(\mathrm{~m}, 2 \mathrm{H}), 4.35(\mathrm{q}, J=6.3 \mathrm{~Hz}, 1 \mathrm{H}), 5.28(\mathrm{~d}, J=10.5 \mathrm{~Hz}, 1 \mathrm{H}), 5.38(\mathrm{~d}, J=17.4 \mathrm{~Hz}, 1 \mathrm{H})$, 5.78-5.95 (m, 1H). ${ }^{13} \mathrm{C} \mathrm{NMR}\left(\mathrm{CDCl}_{3}\right) \delta(\mathrm{ppm}): 25.12,25.19,26.49,27.22,66.22,74.21,75.94,76.38$, 77.42, 80.28, 109.92, 113.85, 119.12, 135.97. HRMS $m / z$ : calcd for $\mathrm{C}_{14} \mathrm{H}_{24} \mathrm{NO}_{4}[\mathrm{M}+\mathrm{H}]^{+}: 270.1705$, found: 270.1699 .

\section{2. (2S, 3S, 4R, 5R)-2-[(R)-2,2-dimethyl-1,3-dioxolan-4-yl]-3,4- isopropylidenedideoxy-5-} ethylpyrrolidine.

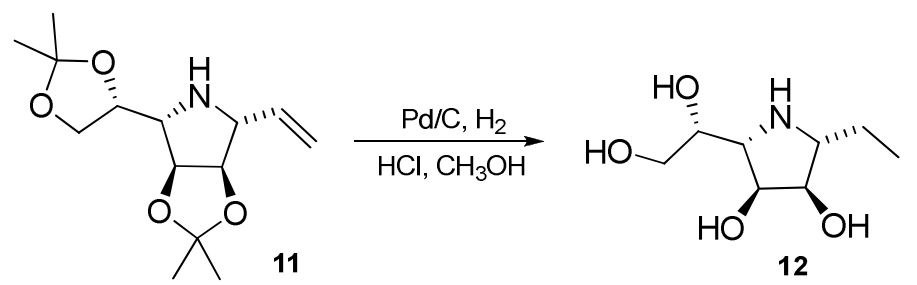

Amine 11 (160 mg, $0.39 \mathrm{mmol}$ ) was dissolved in $30 \mathrm{ml}$ of methanol and 8 drops of concentrated hydrochloric acid. palladium-carbon $(10 \%, 50 \mathrm{mg})$ was then added and the mixture was stirred under hydrogen atmosphere over night. the solid was filter off and the filtration was concentrated to give the desired product $76 \mathrm{mg}$, yield $100 \%$.

$[\alpha]_{\mathrm{D}}^{20}=-9.34\left(\mathrm{c}=1.07 \mathrm{CHCl}_{3}\right), \mathrm{IR} v\left(\mathrm{~cm}^{-1}\right): 3634(\mathrm{br}), 3290(\mathrm{br}), 2937(\mathrm{~m}), 1583(\mathrm{~m}), 1407(\mathrm{~s}), 1349(\mathrm{~m})$, 1328 (m), 1239 (m), 1102 (vs), 1064 (vs), 957 (w); ${ }^{1} \mathrm{H}$ NMR $\left(\mathrm{D}_{2} \mathrm{O}\right) \delta(\mathrm{ppm}): 0.95$ (t, $J=7.3 \mathrm{~Hz}, 3 \mathrm{H}$, $\left.\mathrm{CH}_{3}\right), 1.63-1.76\left(\mathrm{~m}, 2 \mathrm{H}, \mathrm{CH}_{2}\right), 3.36(\mathrm{q}, J=7.1 \mathrm{~Hz}, 1 \mathrm{H}, \mathrm{H}-5), 3.49$ (t, $\left.J=5.9 \mathrm{~Hz}, 1 \mathrm{H}, \mathrm{H}-2\right), 3.56$ (dd, $J$ $=11.8,5.3 \mathrm{~Hz}, 1 \mathrm{H}, \mathrm{H}-5^{\prime}$ ), 3.65 (dd, $\left.J=12.0,3.8 \mathrm{~Hz}, 1 \mathrm{H}, \mathrm{H}-5^{\prime}\right), 3.90$ (dd, $J=9.7,4.4 \mathrm{~Hz}, 1 \mathrm{H}, \mathrm{H}^{\prime} 4^{\prime}$ ), 
3.97 (t, $J=5.3 \mathrm{~Hz}, 1 \mathrm{H}, \mathrm{H}-4), 4.15$ (t, $J=6.0 \mathrm{~Hz}, 1 \mathrm{H}, \mathrm{H}-3) .{ }^{13} \mathrm{C} \mathrm{NMR}\left(\mathrm{D}_{2} \mathrm{O}\right) \delta(\mathrm{ppm}): 9.80\left(\mathrm{CH}_{3}\right), 22.76$

$\left(\mathrm{CH}_{2}\right), 62.85$ (C5'), 63.80 (C-5), 64.63 (C-2), 68.05 (C-4'), 70.51 (C-3), 72.52 (C-4).

HRMS $m / z$ : calcd for $\mathrm{C}_{8} \mathrm{H}_{18} \mathrm{NO}_{4}[\mathrm{M}+\mathrm{H}]^{+}:$192.1236, found:192.1229.

\section{Reference}

[1] Kamm, O. Org. Synth., 1941, Coll. Vol. 1: 445.

[2] Nguyen, T. B.; Martel, A.; Dhal, R.; Dujardin, G. Synthesis 2009, 3174-3176.

[3] Tice, C. M.; Ganem, B. J. Org. Chem. 1983, 48, 5048-5050; (b) Chan, K. S.; Yeung,W.-K.; Chan, R.-J.;Wang, T.-C.; Mak,W. J. Org. Chem. 1995, 60, 1741-1747; (c) Bigdeli, M. A.; Nikje, M. M. A. Monatsh. Chem. 2001, 132, 1547-1549; (d) Y. Fu, Y. Liu, M. Wang, Y. Yang, Y. Chen, K. Cai, Joural Northwest Normal University ( Natural Science), 2011, 47(4), 65-68.

[4] Metzger, A.; Schade, M. A.; Manolikakes, G.; Knochel, P. Chem. Asian J. 2008, 3,1678-1691.

[5] Metzger, A.; Bernhardt, S.; Manolikakes, G.; Knochel, P. Angew. Chem. Int. Ed. 2010, 49, 46654668 


\section{Cover Letter}

Dear Editors and Referees:

I am very glad to submit our manuscript to Organic \& Biomolecular Chemistry. In this manuscript entitled with Trimethylsilyl Chloride Promoted Synthesis of $\alpha$-branched amines by Nucleophilic Addition of Organozinc Halides to Nitrones. we reported a novel TMSCl promoted addition reaction of organozinc halides with $N, \alpha$-diphenyl nitrones. The importance of this work can be viewed as follows:

Nitrones are easily available starting material for the synthesis of structurally variable amines either by 1,3-dipolar cycloaddition reaction or by nucleophilic addition reactions. The nucleophilic addition reaction of Grignards or Organolithium reagents with nitrones are explored widely and used frequently in the synthesis of amines. However, organozinc reagents are rarely used in these areas with the exception of diorganozinc reagents or some diorganozinc induced reactions. The organozinc halides, owing to their low nucleophilicities, are not employed in these reactions to date. We here first report a novel TMSCl promoted addition reaction of organozinc halides with nitrones, in which TMSCI were shown to be both a necessary reactant and a ready hydroxylamine protecting reagent.

With the presence of $\mathrm{TMSCl}$, organozinc halides reacted with nitrones readily and easily in high yield under very mild reaction condition, however, without TMSCl, this type of reaction did not proceed under this condition. Even when the temperature was elevated, the produced hydroxylamines are easily decomposed under air condition, and thus very low yield obtained. Our TMSCl promoted methods have advantages over the tranditional organometallic methods are that:

1. TMSCl is the promotor, thus these reactions can be proceeded under very mild conditions.

2. The obtained hudroxylamines were protected in situ by $\mathrm{TMSCl}$, and thus led to very yields.

3. Oganozinc halides can be highly functionalized, thus our method can be used into construction of highly functionalized molecules. 
The produced O-trimethylsily hydroxylamines can be easily reduced to corresponding amines by a zinc metal in the presence of $\mathrm{Cu}(\mathrm{OAc})_{2}$ in $\mathrm{NH}_{4} \mathrm{Cl}$ solution. 4. This method was shown can be successfully used in natural product synthesis such as in the synthesis of polyhydroxylated pyrrolidines.

Please consider our manuscript and we would greatly appreciate if there are some suggestions on our work.

Best wishes,

Ying Fu

Email: fuying@iccas.ac.cn

College of Chemistry and Chemical Engineering

Northwest Normal University

Lanzhou, An'ning East Road No.967

Gansu Province. 730070

PR China

Telephone: +86-931-7971533

Mobile : +86-13919057389

Fax: +86-931-7971989 
Trimethylsilyl Chloride Promoted Synthesis of $\alpha$-branched amines by Nucleophilic Addition Organozinc Halides to Nitrones

Ying Fu, Yanhua Liu, Yaojuan Chen, Helmut M. Hügel, Danfeng Huang and Yulai Hu

$$
\text { Page - Page }
$$

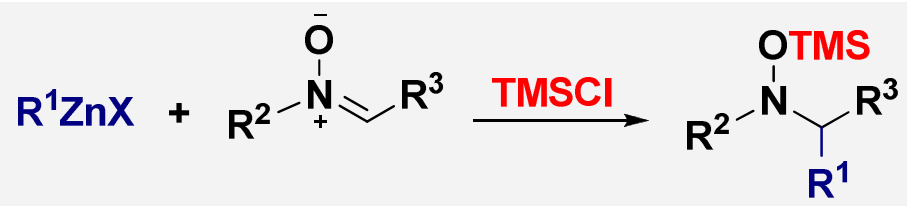

Activation and protection: The activation of nitrone 1,3-dipole is a highly desirable when they were attributed to weak nucleophiles such as organozinc reagents. Gratifying, TMSCl was found to be both an indispensable reaction promoter and a ready hydroxylamine protection reagent in these reactions. 

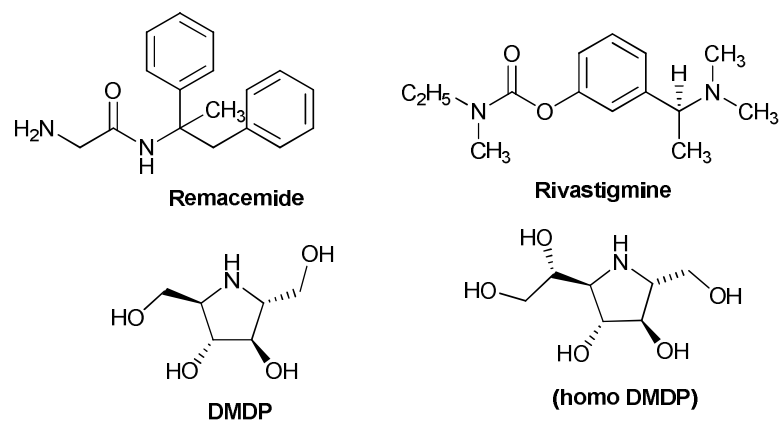

Fig 1. drugs and natural products of $\alpha$-branched amine
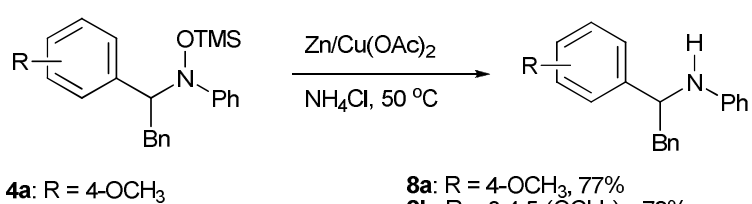

4f: $\mathrm{R}=3,4,5-\left(\mathrm{OCH}_{3}\right)_{3}$,

8b: $\mathrm{R}=3,4,5-\left(\mathrm{OCH}_{3}\right)_{3}, 72 \%$

Scheme 1. Reduction of $O$-TMS hydroxylamine ethers to amines

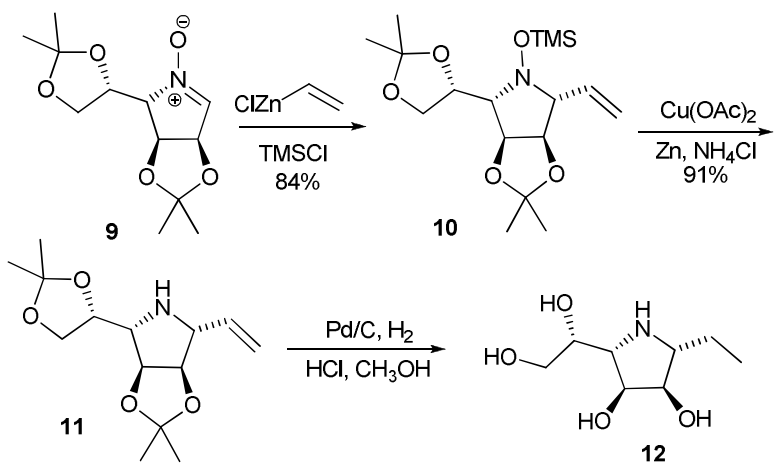

Scheme 2. Synthesis of branched polyhydroxylated pyrrolidine 12 